Large Time Asymptotics in

Contaminant Transport in Porous Media

C.N. Dawson

C.J. van Duijn

R.E. Grundy

November 1994

TR94-39 



\title{
Large Time Asymptotics in Contaminant Transport in Porous media
}

\author{
C.N. Dawson \\ Department of Computational \& Applied Mathematics \\ Rice University \\ P.O. Box 1892 \\ Houston, Texas $77251-1892$ \\ USA \\ C.J. van Duijn * \\ Department of Mathematics \\ Delft University of Teclinology \\ P.O. Box 5031 \\ 2600 GA Delft \\ The Netherlands \\ R.E. Grundy \\ Mathematical Institute \\ University of St. Andrews \\ North Haugh, St. Andrews \\ KY16 9SS \\ Scotland
}

* Corresponding author 



\begin{abstract}
In this paper we derive large time solutions of the partial differential equations modelling contaminant transport in porous media for initial data with bounded support. While the main emphasis is on two space dimensions, for the sake of completeness we give a brief summary of the corresponding results for one space dimension. The philosophy behind the paper is to compare the results of a formal asymptotic analysis of the governing equations as $t \rightarrow \infty$ with numerical solutions of the complete intitial value problem. The analytic results are obtained using the method of "asymptotic balancing" which identifies the dominant terms in the model equations determining the behaviour of the solution in the large time limit. These are found in terms of time scaled space similarity variables and the procedure results in a reduction of the number of independent variables in the original partial differential equation. This generates what we call a reduced equation the solution of which depends crucially on the value of a parameter appearing in the problem. In some cases the reduced equation can be solved explicity while in others they have a particularly intractable structure which inhibits any analytic or numerical progress. However we can extract a number of global and local properties of the solution which enables us to form a reasonably complete picture of what the profiles look like. Extensive comparison with numerical solution of the original initial value problem provides convincing confirmation of our analytic solutions. In the final section of the paper, by way of motivation for the work, we give some results concerning the temporal behaviour of certain moments of the two dimensional profiles commonly used to compute pliysical parameter characteristics for contaminant transport in porous media.
\end{abstract}

\title{
1 Introduction and model
}

In this paper we investigate the large time behaviour of a reactive solute which undergoes equilibrium adsorption in a porous medium and which, at a certain instant in time, is present in the form of a pulse. Describing the adsorption reaction by a Freundlich isotherm, we shall show how the exponent in the isotherm influences the shape of the evolving limit profile. This will be done for pulses extending in one and two space dimensions.

Let us start with a brief description of the underlying transport model in $\mathbb{R}^{2}$. A description in one space variable is then obvious, see also Grundy et al. (1994).

Consider the flow of an incompressible fluid through a homogeneous and saturated porous medium. We assume that the flow is steady, macroscopically one dimensional and directed along what is chosen to be the positive $x$-axis. It is characterized by the specific discharge, which will be denoted by $q(\mathrm{~m} / \mathrm{s})$.

In the fluid a one-species solute is present at tracer level concentration $C\left(\mathrm{~mol} / \mathrm{m}^{3}\right)$. This means that the flow is independent of the solute concentration. We shall therefore take $q$ to be a known positive constant. If no adsorption reactions occur between the solute and the surrounding solid part of the porous medium, then the transport is determined by convection, molecular diffusion and mechanical dispersion, see for instance Bear (1972) 
or Freeze \& Cherry (1979). However, if adsorption reactions do take place, this has to be taken into account when describing the transport process. In this reactive case we denote by $S(\mathrm{~mol} / \mathrm{kg}$ porous material) the adsorbed concentration. If the initial conditions are such that both $C$ and $S$ can be viewed as functions of time $t$ and of the Cartesian space coordinates $x$ and $y$ only, we obtain from mass conservation the equation

$$
\frac{\partial}{\partial t}\{0 C+\rho S\}+q \frac{\partial C}{\partial x}=O D_{x x} \frac{\partial^{2} C}{\partial x^{2}}+0 D_{y y} \frac{\partial^{2} C}{\partial y^{2}}
$$

Here $\theta(-)$ is the porosity of the porous material, $\rho\left(\mathrm{kg} / \mathrm{m}^{3}\right)$ its bulk mass density and $D_{x x}, D_{y y}\left(\mathrm{~m}^{2} / \mathrm{s}\right)$ are coefficients describing hydrodynamic dispersion, which is the sum of molecular diffusion and mechanical dispersion. All coefficients in (1.1) are considered as being constant and positive. The term $\rho \frac{\partial S}{\partial t}$ represents the rate of change of concentration on the porous matrix due to adsorption or desorption.

In this paper we describe the adsorption reactions by means of a Freundlich isotherm. That is we set

$$
S=K C^{p} \quad(p>0),
$$

where $K$ and $p$ are positive constants. An extensive treatment of chemical reactions arising in the transport of solutes through porous media is given by van Duijn \& Knabner (1992), van Duijn et al. (1993) and in a review paper by Weber et al. (1991). Often one finds $0<p \leq 1$ for the Freundlich exponent in (1.2). However Giles et al. (1974) give evidence that $p>1$ also arises.

Combining (1.1) and (1.2) yields the nonlinear diffusion equation

$$
\frac{\partial}{\partial t}\left\{C+\frac{\rho K}{\theta} C^{p}\right\}+v \frac{\partial C}{\partial x}=D_{x x} \frac{\partial^{2} C}{\partial x^{2}}+D_{y y} \frac{\partial^{2} C}{\partial y^{2}}
$$

where $v=q / 0$ denotes the average fluid velocity. We consider solutions of this equation in the set

$$
Q=\{(x, y, t):-\infty<x, y<\infty, t>0\}
$$

subject to the initial condition

$$
C(x, y, 0)=C_{0}(x, y)
$$

at $t=0$. To eliminate the constants from (1.3) we introduce the following redefinitions:

$$
\begin{gathered}
p \neq 1 \begin{cases}u:=\left(\frac{\rho K}{\theta}\right)^{\frac{1}{p-1}} C, & t:=\frac{v^{2}}{D_{x x}} t \\
x:=\frac{v}{D_{x x}} x, & y:=\frac{v}{\sqrt{D_{x x} D_{y y}}} y\end{cases} \\
p=1 \begin{cases}u:=C, & t:=2 \frac{v^{2}}{D_{x x}}\left(1+\frac{\rho K}{\theta}\right)^{-1} \\
x:=\frac{v}{D_{x x}} x, & y:=\frac{v}{\sqrt{D_{x x} D_{y y}} y}\end{cases}
\end{gathered}
$$


This gives the initial value problem (for all $p>0$ )

$$
\begin{aligned}
& (I V P): \quad \frac{\partial}{\partial t}\left(u+u^{p}\right)+\frac{\partial u}{\partial x}=\frac{\partial^{2} u}{\partial x^{2}}+\frac{\partial^{2} u}{\partial y^{2}} \quad \text { for }(x, y, t) \in Q \\
& u(x, y, 0)=u_{0}(x, y) \quad \text { for }(x, y) \in \mathbb{R}^{2}
\end{aligned}
$$

where

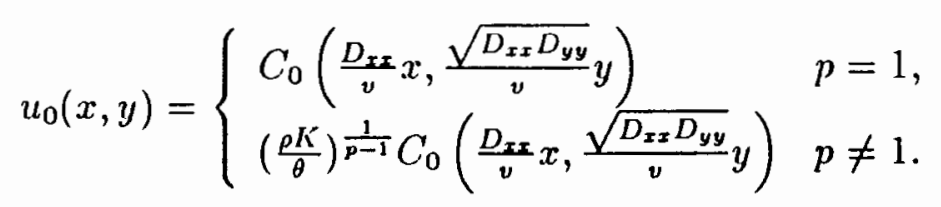

Note that in many cases of practical interest $\frac{\rho K}{\theta}>>1$. This means that the implication of these redefinitions is quite different for $p<1, p=1$ and $p>1$, so one has to bear this in mind when comparing solutions of Problem (IVP) for different values of $p$.

In equation (1.7) one can view the term $\left(1+p u^{p-1}\right)$ as a concentration dependent capacity. For $p \geq 1$ this capacity is bounded for all bounded $u \geq 0$, while for $p<1$ it blows up as $u \searrow 0$. In mathematical terms we say that equation (1.7) is uniformly parabolic when $p \geq 1$ and is degenerate parabolic when $p<1$. The consequence of this is the following. Suppose the initial concentration $u_{0}$ vanishes outside a disc $D_{R}(0)$ (centre at origin, radius $R$ ), i.e. $u_{0}(x, y)=0$ for all $x^{2}+y^{2} \geq R^{2}$. The uniform parabolicity $(p \geq 1)$ implies that $u(x, y, t)>0$ everywhere in $\mathbb{R}^{2}$ for any $t>0$, while the degenerate parabolicity implies that $u(x, y, t)=0$ outside a disc $D_{R(t)}(0)$ having a radius which expands in time $(R<R(t)<\infty$ and $R(t) \rightarrow \infty$ as $t \rightarrow \infty)$. Hence if $p<1$ and depending on the initial distribution, a free boundary may occur which separates the region where $u>0$ from the region where $u=0$. The theory for equation (1.7) in one-space dimension, $u=u(x, t)$, is given by Gilding (1989). The results for higher space dimensions are scattered throughout the mathematics literature, see for instance DiBenidetto (1983). Aronson (1986) presents an interesting survey on the equation without convection.

We want to investigate the large time behaviour of non-negative solutions of Problem (IVP) ( $u \geq 0$, with $u$ the redefined concentration) which satisfy mass conservation: that is, we shall suppose that for all $t \geq 0, u(x, y, t) \rightarrow 0$ sufficiently fast as $|x|,|y| \rightarrow \infty$ so that $u+u^{p}$ is integrable in $\mathbb{R}^{2}$ for all $t \geq 0$.

This implies that

$$
\iint_{\mathbf{R}^{2}}\left(u+u^{p}\right)(x, y, t) d x d y=\iint_{R^{2}}\left(u_{0}+u_{0}^{P}\right)(x, y) d x d y:=M
$$

for all $t \geq 0$.

This invariance property, together with scaling arguments, plays a crucial role in establishing the asymptotic solution. Based on intuition one expects that $u$ will become small for large times. Therefore in this limit for the nondegenerate case $p \geq 1$, one would expect to replace $u+u^{p}$ by $u$ in equation (1.7). This would lead to a linear convection - diffusion equation and consequently to a limit profile which is independent of the Freundlich exponent $p$. However that is not what we observe numerically, see Figures 2.2 and 4.1. 
The correct approach is to first transform to the moving coordinate system

$$
t=t, y=y \text { and } \xi=t-x
$$

which yields the equation

$$
\frac{\partial}{\partial t}\left(u+u^{p}\right)+\frac{\partial u^{p}}{\partial \xi}=\frac{\partial^{2} u}{\partial \xi^{2}}+\frac{\partial^{2} u}{\partial y^{2}} .
$$

In this equation we are now allowed to replace $u+u^{p}$ by $u$ as $t$ becomes large and obtain the nonlinear convection - diffusion equation

$$
\frac{\partial u}{\partial t}+\frac{\partial u^{p}}{\partial \xi}=\frac{\partial^{2} u}{\partial \xi^{2}}+\frac{\partial^{2} u}{\partial y^{2}}
$$

Asymptotic results for equation (1.11) in the range $p>1$ were derived rigorously by Escobedo \& Zuazua (1991) and Escobedo et al. (1993a), where further references are given. Using scaling arguments and the principle of asymptotic balancing in the transformed equation (1.10), that is without neglecting apriori the nonlinearity in the time derivative, we arrive at the same conclusions as Escobedo and others in the above mentioned papers. Briefly the results are as follows. For all $p \geq 1$ and for large times we may replace $u+u^{p}$ by $u$ in (1.10). Moreover

(i) For $p>3 / 2$, diffusion dominates convection. This fact is reflected in the asymptotic form whirh is the radial symmetric fundamental solution ${ }^{1}$ of the heat equation.

(ii) For $p=3 / 2$, diffusion and nonlinear convection balance, and the limit profile is the fundamental solution of equation (1.11).

(iii) For $1<p<3 / 2$, diffusion in the $y$-direction balances convection in the $\xi$-direction. The asymptotic profile now behaves like the fundamental solution of the equation

$$
\frac{\partial u}{\partial t}+\frac{\partial u^{p}}{\partial \xi}=\frac{\partial^{2} u}{\partial y^{2}}
$$

In the degenerate situation where $0<p<1$ rigorous mathematical results concerning the asymptotic behaviour of pulse-type solutions are not yet available. However we can still apply the principle of asymptotic balancing, this time directly to equation (1.7). We conclude that for $0<p<1$ convection in the $x$-direction balances diffusion in the $y$ direction as well as the nonlinear time derivation $\partial u^{p} / \partial t$. As a result, the leading order asympotic form is now given by the fundamental solution of the equation

$$
\frac{\partial u^{p}}{\partial t}+\frac{\partial u}{\partial x}=\frac{\partial^{2} u}{\partial y^{2}} \text {. }
$$

\footnotetext{
${ }^{1}$ By the term fundamental solution of a certain evolution equation, such as (1.10) - (1.13), we mean a solution in $Q$ that satisfies $u(x, y, t) \rightarrow M \delta(x, y)$ as $t \searrow 0$, where $\delta$ the two-dimensional Dirac distribution at the origin
} 
In section 2 we introduce the scalings and the principle of asymptotic balancing to derive the equations for the asymptotic similarity solutions which give the above mentioned fundamental solutions. In section 3 we summarize the results from Grundy et al. (1994) about the large time beliaviour in the one-dimensional case $(u=u(x, t))$. There the principle of asymptotic balancing leads to explicit expressions for the limit profiles. Next in section 4 we return to the two-dimensional situation, to study the behaviour of the asymptotic similarity solutions for $p$ in the range $0<p<1$ and $p>1$. In all three sections 2,3 and 4 we present numerical results to support the analytical findings and conjectures. Finally in section 5 we give an interpretation of the asymptotic results. In particular we investigate in what way the movement of the centre of mass of a contaminant pulse and its longitudinal and lateral spreading are influenced by the value of the Freundlich exponent $p$.

We note that the asymptotic balancing procedure can also be carried out for the transport problems in three space dimensions. Escobedo and co-authors also have theoretical results for $p>1$. However the task of obtaining numerically asymptotic solutions of the full problem, namely by solving (1.7) and (1.8) in $\mathbb{R}^{3}$ for large time, is computationaly expensive. Analyzing the corresponding equations for the asymptotic similarity solutions is also difficult. For these reasons we leave the three-dimensional problem for later study.

Up to now we have not mentioned the linear case $p=1$. Here the asymptotic form is given by the fundamental solution of the linear diffusion-convection equation

$$
2 \frac{\partial u}{\partial t}+\frac{\partial u}{\partial x}=\frac{\partial^{2} u}{\partial x^{2}}+\frac{\partial^{2} u}{\partial y^{2}}
$$

namely

$$
u(x, y, t) \rightarrow \frac{M}{4 \pi t} \exp \left\{-\frac{1}{2 t}\left(\left(x-\frac{t}{2}\right)^{2}+y^{2}\right)\right\}
$$

as $t \rightarrow \infty$. Going back to the original variables, see (1.6), we find that the factor 2 will disappear from the exponential to be replaced as usual by an appropriate retardation factor, see for instance Freeze \& Cherry (1979).

\section{Asymptotic balancing}

In this section we introduce and develop the principle of asymptotic balancing as applied to solutions of Problem (IVP). As was explained in the introduction, the nature of the solution to the transport equation (1.7) is quite different for $p>1$ and for $p<1$. Consequently the ansatz about the behaviour of the limiting solutions will be different. We therefore treat these cases separately.

\subsection{The case $p>1$}

The starting point is the translated equation (1.10). The ansatz about the large time behaviour is that we expect the spread of the solution to be incorporated by using the 
similarity variables

$$
\eta=\frac{\xi}{t^{\beta}}=\frac{t-x}{t^{\beta}}, \zeta=\frac{y}{t^{\delta}}
$$

where $\beta, \delta \geq 0$, together with the change of dependent variable

$$
u(\xi, y, t)=t^{\alpha} v(\eta, \zeta, t)
$$

with $\alpha<0$ to simulate temporal decay. That is to say, we expect

$$
v(\eta, \zeta, t)=v_{0}(\eta, \zeta)+o(1) \text { as } t \rightarrow \infty \text {. }
$$

giving

$$
t^{\alpha} v_{0}\left(\frac{t-x}{t^{\beta}}, \frac{y}{t^{\delta}}\right)
$$

as the evolving asymptotic profile. The function $v_{0}$, or rather the equation for $v_{0}$, will result from the asymptotic balancing of the various terms in the $v$-equation. This latter equation follows from (1.10) after substituting the scalings (2.1). The result is the following lengthy expression

$$
\begin{aligned}
& \left(t \frac{\partial}{\partial t}+\alpha-\beta \eta \frac{\partial}{\partial \eta}-\delta \zeta \frac{\partial}{\partial \zeta}\right)\left(v+t^{\alpha(p-1)} v^{p}\right)+ \\
& t^{1+\alpha(p-1)-\beta} \frac{\partial v^{p}}{\partial \eta}=t^{1-2 \beta} \frac{\partial^{2} v}{\partial \eta^{2}}+t^{1-2 \delta} \frac{\partial^{2} v}{\partial \zeta^{2}}
\end{aligned}
$$

Next we recall that we are looking for nonnegative solutions satisfying mass conservation (1.9). For the scaled solution $v$ this gives

$$
M=\iint_{\mathbf{R}^{2}}\left\{t^{\alpha+\beta+\delta} v+t^{\alpha p+\beta+\delta} v^{p}\right\} d \eta d \zeta .
$$

Hence for $p>1$ and $\alpha<0$ we have

$$
M \sim t^{\alpha+\beta+\delta} \iint_{\mathbf{R}^{2}} v_{0} d \eta d \zeta \text { as } t \rightarrow \infty,
$$

and mass invariance requires

$$
\alpha+\beta+\delta=0
$$

We now proceed with the various balancing possibilities in (2.4). First we take the combination(1),(3)and(4), that is the time derivative and the diffusion terms in equation (1.10). Bearing in mind that the term involving $v^{p}$ is small, this balance is only possible if

$$
\beta=\delta=\frac{1}{2}
$$

and

$$
1+\alpha(p-1)-\beta<0
$$


Combining (2.6) - (2.8) gives

$$
\alpha=-1 \text { and } p>3 / 2 \text {. }
$$

For these values of the parameters we substitute expansion (2.2) into equation (2.4). Collecting leading order terms gives the reduced equation

$$
v_{0}+\frac{\eta}{2} \frac{\partial v_{0}}{\partial \eta}+\frac{\zeta}{2} \frac{\partial v_{0}}{\partial \zeta}+\frac{\partial^{2} v_{0}}{\partial \eta^{2}}+\frac{\partial^{2} v_{0}}{\partial \zeta^{2}}=0, \text { for }(\eta, \zeta) \in \mathbb{R}^{2},
$$

with

$$
v_{0} \geq 0 \text { and } \iint_{\mathbb{R}^{2}} v_{0} d \eta d \zeta=M
$$

The solution to this problem is explicity known as

$$
v_{0}(\eta, \zeta)=\frac{M}{4 \pi} e^{-\left(\eta^{2}+\zeta^{2}\right) / 4}
$$

Hence for $p>3 / 2$ we have obtained the asymptotic result

$$
u(x, y, t) \rightarrow t^{-1} v_{0}\left(\frac{t-x}{t^{1 / 2}}, \frac{y}{t^{1 / 2}}\right) \text { as } t \rightarrow \infty .
$$

in which we recognize the fundamental solution of the heat equation.

Note that this profile is radially symmetric with respect to the moving coordinates $(x=t, y=0)$, a behaviour which can be observed from Figure 2.1, showing ievel curves of the numerical solution of Problem (IVP). Ilere the initial condition for $u$ is a pulse, that is $u_{0}=1$ on a square of side 2 centered at the origin and $u_{0}=0$ elsewhere. The chemical reactions are characterized by the Freundlich exponent $p=3$.

Continuing with the principle of asymptotic balancing we now take all terms in equation (2.4). This balance is only possible if (2.7) holds together with

$$
1+\alpha(p-1)-\beta=0,
$$

which gives the borderline case $p=3 / 2$. Proceeding as before we now obtain the reduced equation

$$
v_{0}+\frac{\eta}{2} \frac{\partial v_{0}}{\partial \eta}+\frac{\zeta}{2} \frac{\partial v_{0}}{\partial \zeta}-\frac{\partial v_{0}^{3 / 2}}{\partial \eta}+\frac{\partial^{2} v_{0}}{\partial \eta^{2}}+\frac{\partial^{2} v_{0}}{\partial \zeta^{2}}=0 \text { for },(\eta, \xi) \in \mathbb{R}^{2}
$$

where again $v_{0}$ satisfies (2.11). No closed form solutions are known for this problem. However it has received attention in the mathematical literature. In a recent paper, Aguirre et al. (1990) showed existence and uniqueness (for any given $M>0$ ) of solutions that decay exponentially to zero as $|\zeta|,|\eta| \rightarrow \infty$. These solutions are symmetric in $\zeta$ but not in $\eta$. This behaviour can also be seen in the computations. Figure 2.2 shows the spreading of contaminant, that is the numerical solution of Problem (IVP) with $p=3 / 2$, starting again from a pulse at $t=0$.

Thus for $p=3 / 2$ the asymptotic result is as in (2.13) where now $v_{0}$ satisfies $(2.15)$, (2.11). 


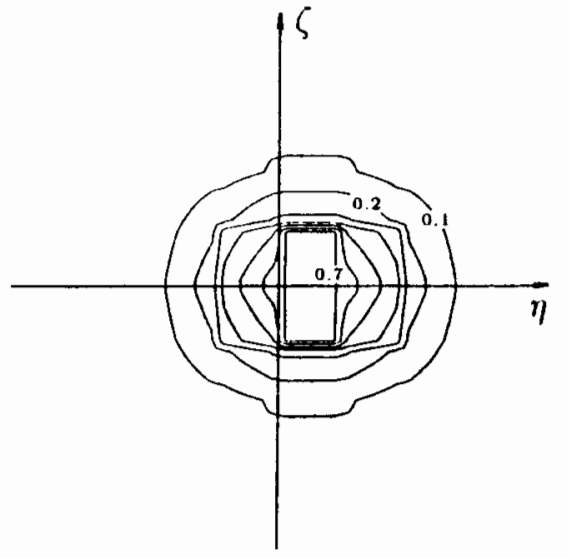

$t=1$

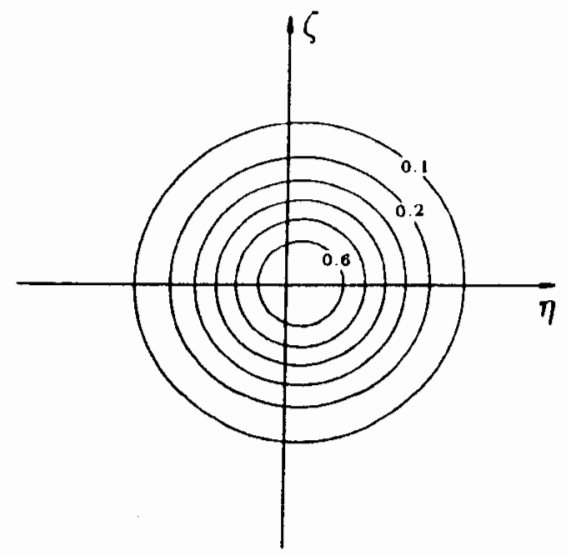

$t=15$

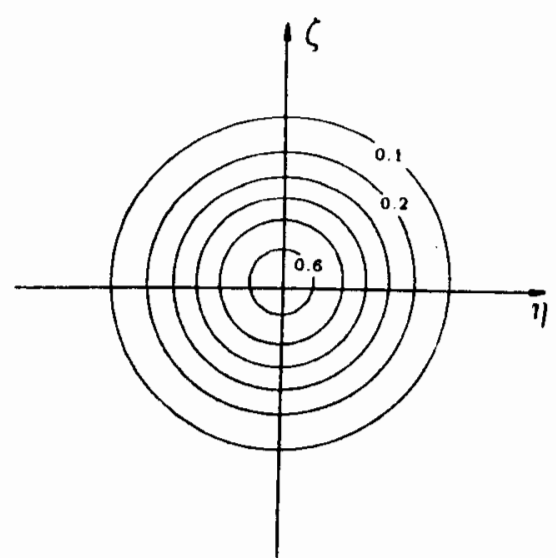

$t=100$

Fig. 2.1: Numerical solution of Problem (IVP) in the scaled variables $v=t u$ and $\eta=$ $(t-x) / t^{1 / 2}, \zeta=y / t^{1 / 2}$. Here $p=3$ and $M=S$ (initially $u_{0}=1$ on the square of side 2 with centre at origin, $u_{0}=0$ elsewhere). Plotled are level curves of $v$ at early $(t=1)$, intermediate $(t=15)$ and final $(t=100)$ times.

In this case the limit profile is the fundamental solution of equation (1.11).

Returning to the balancing of terms in equation (2.4) we check the two remaining possibilities. One is that the $\eta$-diffusion balances the $\eta$-convection; namely the combination (1),(2)and(3). This yields

$$
\beta=\frac{1}{2}, \quad 1+\alpha(p-1)-\beta=0
$$

giving

$$
\alpha=-\frac{1}{2(p-1)}
$$

with $1-2 \delta<0$. Combining these relations with (2.6) results in

$$
\delta=\frac{2-p}{2(p-1)} \text { and } p<3 / 2 \text {. }
$$

The $v_{0}$-equation now becomes

$$
\alpha v_{0}-\beta \eta \frac{\partial v_{0}}{\partial \eta}-\delta \zeta \frac{\partial v_{0}}{\partial \zeta}+\frac{\partial v_{0}^{p}}{\partial \eta}-\frac{\partial^{2} v_{0}}{\partial \eta^{2}}=0
$$

We show that the only nonnegative solution of this equation is the trivial solution $v_{0}=0$. Suppose this is not true and take $v_{0} \geq 0\left(v_{0} \not \equiv 0\right)$ with

$$
m(\zeta)=\int_{-\infty}^{+\infty} v_{0}(\eta, \zeta) d \eta \geq 0
$$



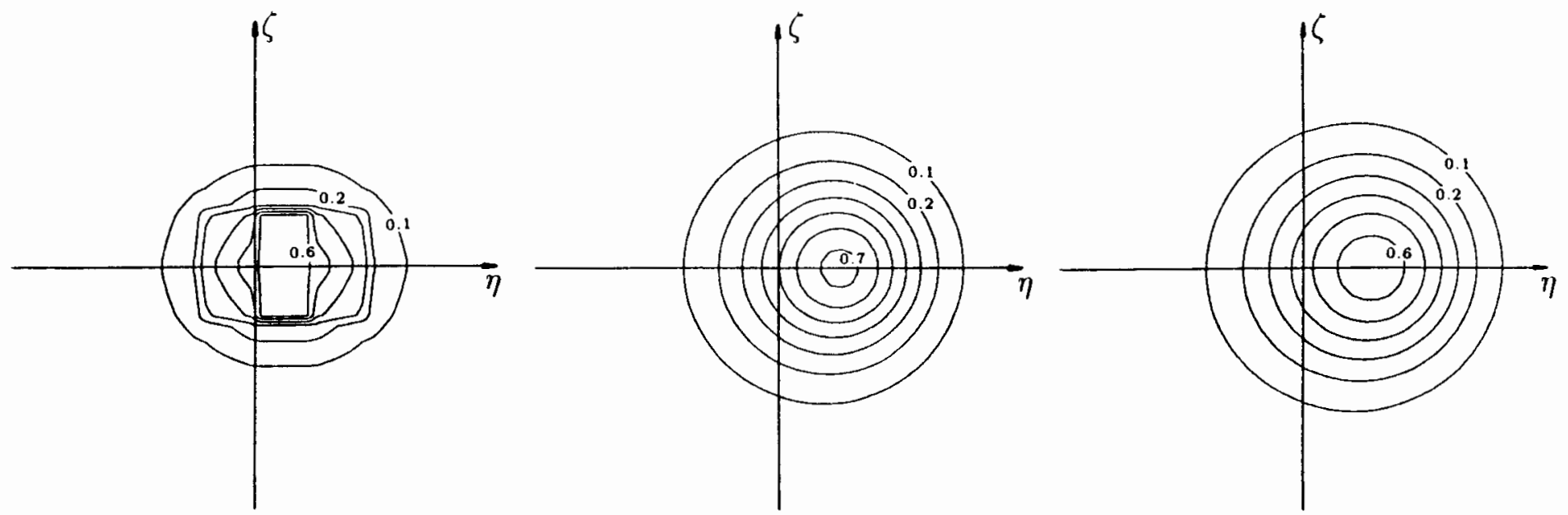

$$
t=1
$$

$$
t=15
$$

$t=100$

Fig. 2.2: Numerical results of Problem (IVP) in the scaled variables, see also Figure 2.1. Here $p=3 / 2$ and $M=8$. The results are given for $t=1, t=15$ and $t=100$.

Integrating all terms in equation (2.16) with respect to $\eta$, and using integration by parts in the second term, results in

$$
(\alpha+\beta) m-\delta \zeta \frac{d m}{d \zeta}=0
$$

or with (2.6)

$$
\zeta \frac{d m}{d \zeta}=-m \leq 0,-\infty<\zeta<\infty .
$$

We deduce from this equation that $m(0)=0$ and $\frac{d m}{d \zeta} \leq 0$ for $\zeta>0$ and $\frac{d m}{d \zeta} \geq 0$ for $\zeta<0$, consequently $m(\zeta) \leq 0$ for all $-\infty<\zeta<\infty$. Equation (2.17) now implies $m(\zeta)=0,-\infty<\zeta<\infty$, and since $v_{0} \geq 0$, that $v_{0}(\eta, \zeta)=0$ for all $(\eta, \zeta) \in \mathbb{R}^{2}$. However this contradicts the mass-invariance and so we reject this combination.

The remaining possible balance is between $\zeta$-diffusion and $\eta$-convection, that is the combination (1), (2)and(4). This gives

$$
\delta=\frac{1}{2}, \quad 1+\alpha(p-1)-\beta=0
$$

and

$$
1-2 \beta<0
$$

Combining these with (2.6) yields

$$
\alpha=-\frac{3}{2 p}, \beta=\frac{3-p}{2 p} \text { and } p<3 / 2 \text {. }
$$


As the reduced $v_{0}$-equation we have

$$
\frac{3}{2 p} v_{0}+\frac{3-p}{2 p} \eta \frac{\partial v_{0}}{\partial \eta}+\frac{\zeta}{2} \frac{\partial v_{0}}{\partial \zeta}-\frac{\partial v_{0}^{p}}{\partial \eta}+\frac{\partial^{2} v_{0}}{\partial \zeta^{2}}=0 \text { for }(\eta, \zeta) \in \mathbb{R}^{2}
$$

where again $v_{0}$ satisfies (2.11). This problem will be considered in some detail in section 4 , but we conclude this section with the observation that for $1<p<3 / 2$ the asymptotic result is

$$
u(x, y, t) \rightarrow t^{-\frac{3}{2 p}} v_{0}\left(\frac{t-x}{t^{\frac{3-p}{2 p}}}, \frac{y}{t^{1 / 2}}\right) \text { as } t \rightarrow \infty,
$$

where $v_{0}$ is the solution of $(2.22),(2.11)$. Numerical evidence for this convergence will be given in section 4 . Note that here the limiting profile (2.23) is the fundamental solution of equation (1.12).

\subsection{The case $0<p<1$}

For this range of values of $p$ we start the analysis directly from equation (1.7). Now the ansatz is that the asymptotic profile can be obtained by introducing the similarity variables

$$
\eta=\frac{x}{t^{b}}, \zeta=\frac{y}{t^{d}}
$$

with $b, d \geq 0$, and the scaling

$$
u(x, y, t)=t^{a} v(\eta, \zeta, t)
$$

where again $a<0$ to incorporate temporal decay. Then as in (2.2), (2.3), we expect

$$
t^{a} v_{0}\left(\frac{x}{t^{b}}, \frac{y}{t^{d}}\right)
$$

to be the asymptotic profile.

As far as mass invarance is concerned we have from (2.4), with $\alpha, \beta, \delta$ replaced by $a, b, d$ respectively, and remembering $p<1$ and $a<0$, that

$$
M \sim t^{a p+b+d} \iint_{\mathbb{R}^{2}} v_{0}^{p} d \eta d \zeta \text { as } t \rightarrow \infty
$$

which implies

$$
a p+b+d=0 \text {. }
$$

If we now substitute (2.25) into equation (1.7) we obtain the $v$-equation

$$
\begin{gathered}
\left(t \frac{\partial}{\partial t}+a p-b \eta \frac{\partial}{\partial \eta}-d \zeta \frac{\partial}{\partial \zeta}\right)\left(t^{a(1-p)} v+v^{p}\right)+ \\
t^{1+a(1-p)-b} \frac{\partial v}{\partial \eta}=t^{1+a(1-p)-2 b} \frac{\partial^{2} v}{\partial \eta^{2}}+t^{1+a(1-p)-2 d} \frac{\partial^{2} v}{\partial \zeta^{2}}
\end{gathered}
$$


Observing that the term involving $t^{a(1-p)} v$ in $l$ is always small, we consider the following combinations. First a four term balance as $t \rightarrow \infty$ gives

$$
b=d=0 \text { and } a=\frac{-1}{1-p}
$$

but does not satisfy $(2.2 S)$ so we reject this possibility.

Next if(1),(2)and(3)balance as $t \rightarrow \infty$, then

$$
b=0, a=\frac{-1}{1-p} \text { and (from (2.28)) } d=\frac{p}{1-p} .
$$

Using these parameter values in equation (2.29) and substituting expansion (2.2), yields the $v_{0}$ equation

$$
-\frac{p}{1-p} v_{0}^{p}-\frac{p}{1-p} \zeta \frac{\partial v_{0}^{p}}{\partial \zeta}+\frac{\partial v_{0}}{\partial \eta}=\frac{\partial^{2} v_{0}}{\partial \eta^{2}}
$$

A similar analysis to the one we used on equation (2.16), shows that the only nonnegative solution is the trivial solution $v_{0}=0$, which contradicts mass invariance. Hence this combination is also rejected. The only remaining possibility is that(1), (2)and(4)alance for large times, that is $\eta$ (or $x$ )-convection balances $\zeta$ (or $y$ )-diffusion. We now find that

$$
1+a(1-p)-b=1+a(1-p)-2 d=0,
$$

which, together with (2.28), yields

$$
a=\frac{-3}{3-p}, b=\frac{2 p}{3-p} \text { and } d=\frac{p}{3-p} .
$$

For the leading order term $v_{0}$ in the large time expansion (2.2) we now have the reduced $v_{0}$-equation

$$
\frac{3 p}{3-p} v_{0}^{p}+\frac{2 p}{3-p} \eta \frac{\partial v_{0}^{p}}{\partial \eta}+\frac{p}{3-p} \zeta \frac{\partial v_{0}^{p}}{\partial \zeta}-\frac{\partial v_{0}}{\partial \eta}+\frac{\partial^{2} v_{0}}{\partial \zeta^{2}}=0 \text { for }(\eta, \zeta) \in \mathbb{R}^{2},
$$

where in addition $v_{0}$ satisfies

$$
v_{0} \geq 0 \text { and } \iint_{R^{2}} v_{0}^{p} d \eta d \zeta=M .
$$

As in the case of equation (2.22), we are dealing here with a nonlinear partial differential equation in two space dimensions. In general such equations cannot be expected to be solved explicity. Nevertheless, we are able to make a number of important observations about the qualitative behaviour of the solutions. We postpone this analysis to section 4 , but merely conclude here that in the range $0<p<1$, the asymptotic behaviour is given by

$$
u(x, y, t) \rightarrow t^{-\frac{3}{3-p}} v_{0}\left(\frac{x}{t^{\frac{2 p}{3-p}}}, \frac{y}{t^{\frac{p}{3-p}}}\right) \text { as } t \rightarrow \infty .
$$

where $v_{0}$ is the solution of problem $(2.32),(2.33)$. Here the asymptotic limit is the fundamental solution of equation (1.13). Also in this case numerical evidence for the convergence will be given in section 4 . 


\section{One dimensional results}

Before we investigate the nature of the solutions of the reduced equations that were derived in the previous section, we want to summarize here some of the asymptotic results for the transport of reactive solutes in one space dimension. These results were recently published by Grundy et al. (1994).

Using the fact that now $u=u(x, t)$ only, we arrive at the one-dimensional initial value problem

$$
\begin{aligned}
\left(I V P_{1}\right): \quad \frac{\partial}{\partial t}\left(u+u^{p}\right)+\frac{\partial u}{\partial x} & =\frac{\partial^{2} u}{\partial x^{2}} \text { for }-\infty<x<\infty, t>0 \\
u(x, 0) & =u_{0}(x) \text { for }-\infty<x<\infty
\end{aligned}
$$

Further we suppose that for all $t \geq 0, u(x, t) \rightarrow 0$ sufficiently fast as $|x| \rightarrow \infty$, so that again the mass-invariance condition

$$
\int_{\mathbb{R}}\left\{u+u^{p}\right\}(x, t) d x=\int_{\mathbb{R}}\left\{u_{0}+u_{0}^{p}\right\}(x) d x:=M
$$

holds for all $t \geq 0$.

To classify the asymptotic behaviour, we distinguish again between the cases $p>1$ and $p<1$. For $p>1$ we introduce the moving coordinates $t=t, \xi=t-x$, and arrive at the transformed equation

$$
\frac{\partial}{\partial t}\left(u+u^{p}\right)+\frac{\partial u^{p}}{\partial \xi}=\frac{\partial^{2} u}{\partial \xi^{2}}
$$

By applying the principle of asymptotic belancing to this equation we find for $p>1$ that uniformly for large time we may replace $u+u^{p}$ in the time derivative by $u$. Thus we may apply the principle to the simplified equation.

$$
\frac{\partial u}{\partial t}+\frac{\partial u^{p}}{\partial \xi}=\frac{\partial^{2} u}{\partial \xi^{2}} \quad \text { for },-\infty<\xi<\infty, t>0
$$

from which, under the constant mass constraint (3.3), we obtain the following results.

For $p>2$, the diffusion dominates the convection as $t \rightarrow \infty$. As a result the limiting profile is the fundamental solution of the heat equation, which is symmetric with respect to the moving coordinate $x=t$ and we find

$$
u(x, t) \rightarrow \frac{M}{2 \sqrt{\pi t}} \exp \left\{-(x-t)^{2} / 4 t\right\} \text { as } t \rightarrow \infty .
$$

This result was also verified numerically. It is illustrated in Figure 3.1 which gives $t^{1 / 2} u$ as a function of the similarity variable $(t-x) / t^{1 / 2}$ for $p=3$ and the initial data

$$
u_{0}(x)=H(x+1)-H(x-1),-\infty<x<\infty,
$$

where $H$ is the Heaviside function. This gives $M=4$. 


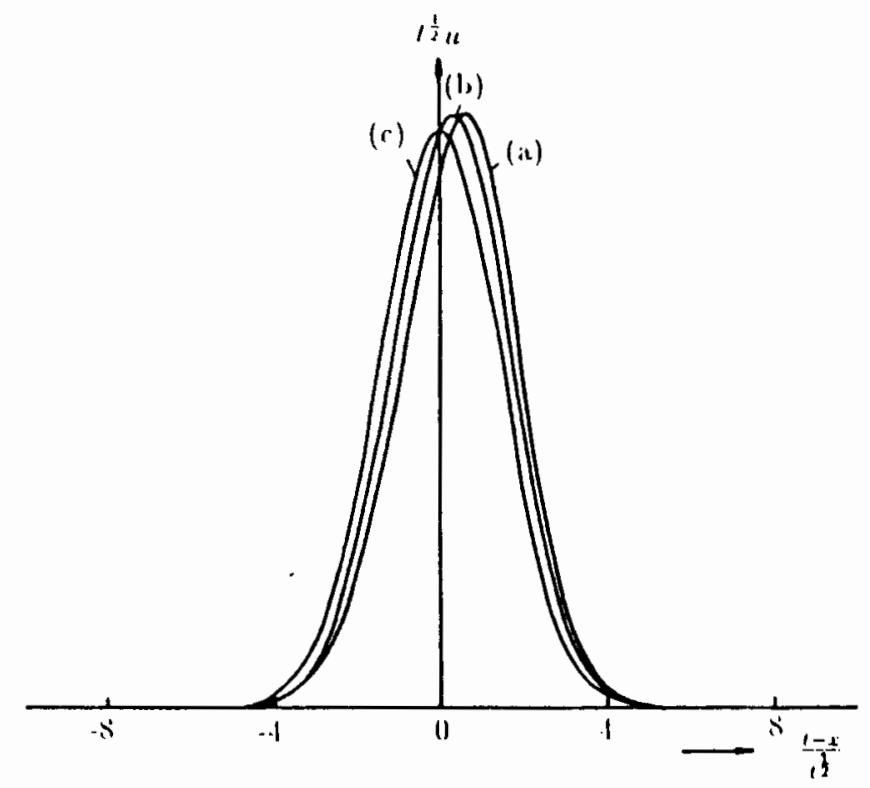

Fig. 3.1: Convergence of the numerical solution of Problem (IV'P $)$ for $p=3$ and $u_{0}$ given by (3.7). Here $t^{1 / 2} u$ is plotted as a function of $(t-x) / t^{1 / 2}$ for $(a) t=40$, (b) $t=1000$ and (c) the limiting profile (3.6).

For $p=2$, the diffusion balances the convection as $t \rightarrow \infty$. Now the limiting profile is the fundamental solution of Burgers equation (equation (3.5) with $p=2$ ), which is right asymmetric with respect to $x=t$. The large time behaviour is

$$
u(x, t) \rightarrow \frac{1}{\sqrt{\pi t}} \frac{\exp \left(-\eta^{2} / 4\right)}{\{A+\operatorname{erf}(\eta / 2)\}} \text { as } t \rightarrow \infty,
$$

where

$$
\eta=(t-x) / t^{1 / 2} \text { and } A=\left(e^{M}+1\right) /\left(e^{M}-1\right) .
$$

Again we verified the convergence numerically and the result is given in Figure 3.2. We note here that in both the cases $p=3$ and $p=2$ convergence to the final profile can be accelerated by using strained coordinates to incorporate the leading error term. See Grundy et al. (1994).

For $1<p<2$, convection dominates diffusion as $t \rightarrow \infty$ and the asymptotic profile is the solution of the hyperbolic problem,

$$
\begin{gathered}
\frac{\partial u}{\partial t}+\frac{\partial u^{p}}{\partial \xi}=0 \quad \text { for }-\infty<\xi<\infty, t>0, \\
u(\xi, 0)=M \delta(\xi) \text { for }-\infty<\xi<\infty,
\end{gathered}
$$

where $\delta$ denotes the one dimensional Dirac distribution at the origin. The solution of this problem can be found in terms of the similarity variables arising from the asymptotic balancing. The convergence result then becomes

$$
u(x, t) \rightarrow t^{-1 / p} v_{0}\left(\frac{t-x}{p t^{1 / p}}\right) \quad \text { as } t \rightarrow \infty,
$$




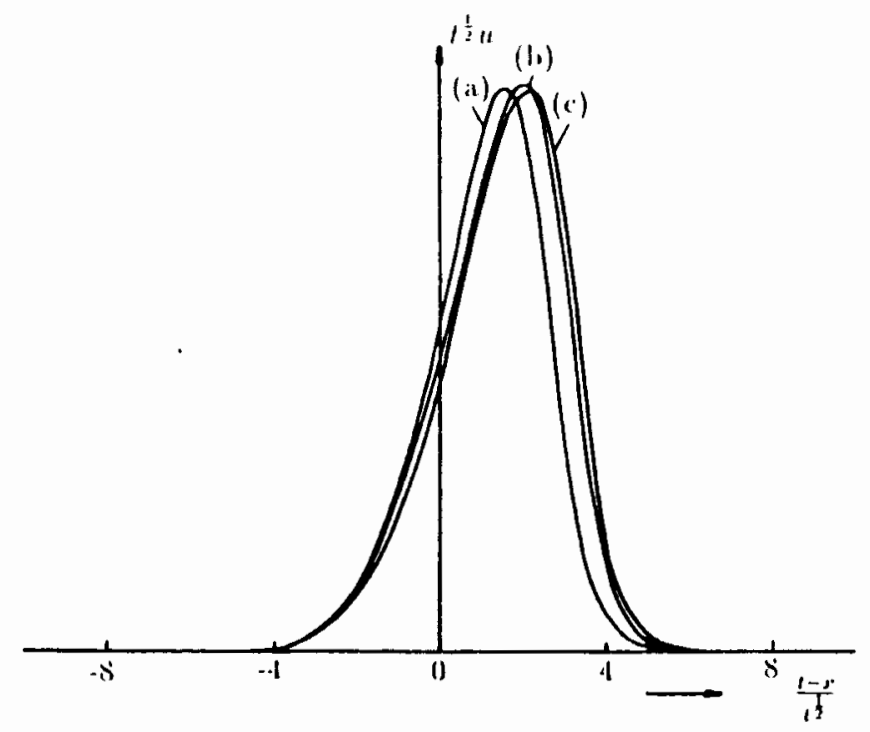

Fig. 3.2: Convergence of the numcrical solution of Problem $\left(I V P_{1}\right)$ for $p=2$ and $u_{0}$ given by (3.7). Again $t^{1 / 2} u$ is plotted as a function of the similarity variable $\eta$ for (a) $t=40$, (b) $t=1000$ and (c) the limiting profile (3.8).

where

$$
v_{0}\left(\frac{\eta}{p}\right)= \begin{cases}0 & \eta \leq 0 \\ \left(\frac{\eta}{p}\right)^{\frac{1}{p-1}} & 0<\eta<\eta_{1} \\ 0 & \eta \geq \eta_{1}\end{cases}
$$

with

$$
\eta_{1}=p\left(\frac{M}{p-1}\right)^{\frac{p-1}{p}} .
$$

Note that this limiting profile is discontinuous along the curve $x=t-\eta_{1} t^{1 / p}$ and vanishes identically for $x \geq t$ and $x<t-\eta_{1} t^{1 / p}$. In the asymptotic jargon one calls (3.12) the zeroth order outer solution. Grundy et al. (1994) also investigated the bounclary layers near $\eta=0$ and $\eta=\eta_{1}$, at the leading and the trailing edge of the profile. Figure 3.3 shows the numerical convergence for $p=1.5$ and again $u_{0}$ given by (3.7). Since the convergence was quite slow we accelerated the process by modifying equation (3.1) into

$$
\frac{\partial}{\partial t}\left(u+u^{p}\right)+\frac{\partial u}{\partial x}=\varepsilon \frac{\partial^{2} u}{\partial x^{2}}
$$

with $\varepsilon$ small.

We observe that the case $p=2$, where all terms in the equation balance, can be viewed as borderline, in the same way as $p=3 / 2$ is in the two dimensional problem. If one considered the problem in $N$ space dimensions, one would find $p=1+\frac{1}{N}$ to be the borderline case, see also Escobedo (1993b). 


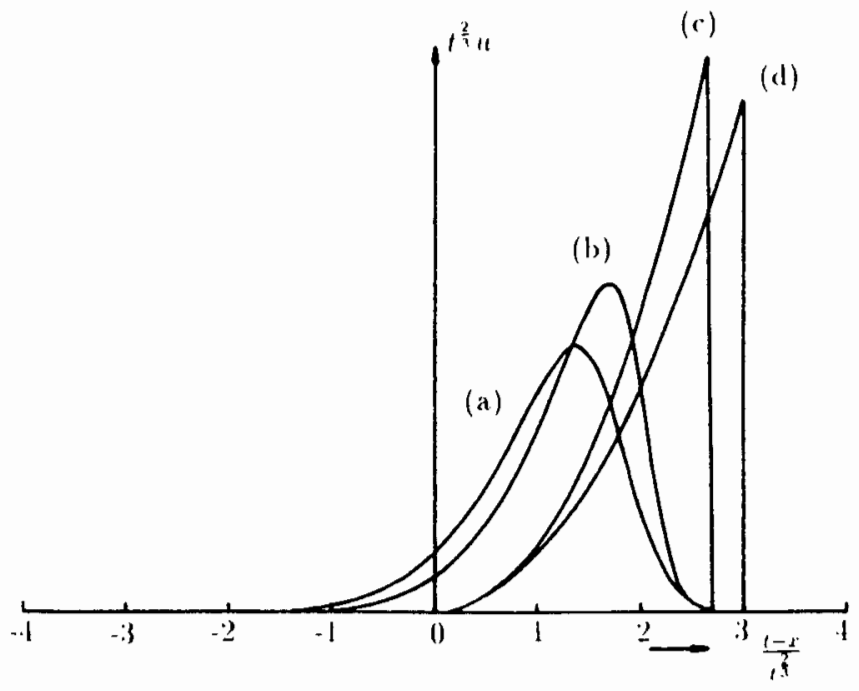

Fig. 3.3: Convergence of the numerical solution of (3.14), (3.2) for $p=1.5, u_{0}$ given by (3.7) and $\varepsilon=10^{-2}$. Here $t^{\frac{1}{p}} u$ is plotted against the similarity variable $\eta=(t-x) / t^{1 / p}$ for (a) $t=80$ (b) $t=320$ (c) $t=4000$ and (d) the zeroth order outer solution (3.12).

We conclude this section with the asymptotic results for the case $0<p<1$. Here the method of asymptotic balancing is applied directly to equation (3.1) from which we deduce that $x$-convection dominates $x$-difusion. Consequently the limiting profile is the solution of the hyperbolic problem

$$
\begin{gathered}
\frac{\partial u^{p}}{\partial t}+\frac{\partial u}{\partial x}=0 \text { for }-\infty<x<\infty, t>0, \\
u^{p}(x, 0)=M \delta(x) \text { lor }-\infty<x<\infty .
\end{gathered}
$$

In terms of the similarity variables arising from the asymptotic balancing we have the convergence result

$$
u(x, t) \rightarrow t^{-1} v_{0}\left(\frac{p x}{t^{p}}\right) \text { as } t \rightarrow \infty
$$

where now

$$
v_{0}(p \eta)= \begin{cases}0 & \eta \leq 0 \\ (p \eta)^{\frac{1}{1-p}} & 0<\eta<\eta_{2} \\ 0 & \eta>\eta_{2}\end{cases}
$$

with

$$
\eta_{2}=p^{-p}\left(\frac{M}{1-p}\right)^{1-p}
$$

Here too the asymptotic limit is discontinuous, now along the curve $x=\eta_{2} t^{p}$, and vanishes identically for $x \leq 0$ and $x>\eta_{2} t^{p}$. Boundary layers can again be inserted near the leading 
and trailing edges of this zero order outer solution, a task porformed in the paper by Grundy et al. (1994). Figure 3.4 shows the convergence of the numerical solution for $p=0.5$ and $u_{0}$ given by (3.7). Also here the convergence (3.16) was slow and to avoid excessive computing times we solved the modified initial value problem (3.14), (3.2) with $\varepsilon=10^{-2}$.

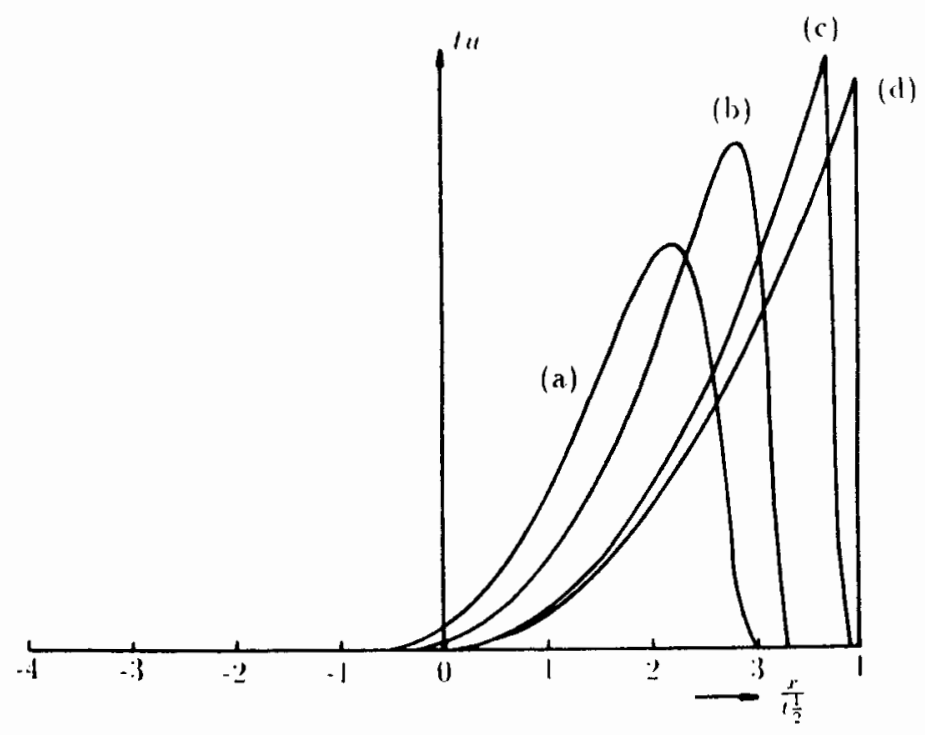

Fig. 3.4: Solution of (3.14), (3.2) converging toward the asymptotic limit for $p=0.5, u_{0}$ as in (3.7) and $\varepsilon=10^{-2}$. The scaled function tu is plotted against the similarity variable $\eta=x t^{-p}$ for (a) $t=80$, (b) $t=320$ and (c) $t=2000$ and (d) the zeroth order outer solution (3.18).

\section{The reduced equations in two dimensions}

In section 2 we saw that the principle of asymptotic balancing leads to partial differential equations, namely, the reduced $v_{0}$-equations, in the scaled space variables. If these reduced equations could be solved, or otherwise if properties of the solutions were known, then a precise characterization of the limiting profiles could be given.

In this respect the ranges $1<p<3 / 2$ and $0<p<1$ need further attention, because in both cases the reduced equations are complicated nonlinear equations which do not admit closed form solutions. In this section we shall analyse the structure of the solutions of these equations without solving them explicitly. Again we treat the cases $1<p<3 / 2$ and $0<p<1$ separately. 


\subsection{The reduced equation for $1<p<3 / 2$}

The problem to be considered is the following. We have to find a function $v_{0}$, such that

$$
\frac{3}{2 p} v_{0}+\frac{3-p}{2 p} \eta \frac{\partial v_{0}}{\partial \eta}+\frac{\zeta}{2} \frac{\partial v_{0}}{\partial \zeta}-\frac{\partial v_{0}^{p}}{\partial \eta}+\frac{\partial^{2} v_{0}}{\partial \zeta^{2}}=0
$$

for all $(\eta, \zeta) \in \mathbb{R}^{2}$, and

$$
v_{0} \geq 0, \quad \iint_{\mathbb{R}^{2}} v_{0} d \eta d \zeta=M
$$

Before cliscussing some properties of the solutions for $v_{0}$, we observe that equation (4.1) is second order in $\zeta$, but only first order in $\eta$. This means that the profiles will have less smoothness in $\eta$-direction, the direction of flow, than in the transverse $\zeta$-direction.

Equation (4.1) was discussed in a recent paper by Escobedo et al. (1993a). In that paper they study the large time behaviour of solutions of the initial value problem for equation (1.11), with $1<p<3 / 2$, where $\iint_{\mathbb{R}^{2}} u_{0}=M$. They show convergence to a fundamental solution of equation (1.12), which has diffusion only in the transverse direction to the flow. Moreover they show that this fundamental solution, parametrized by $M>0$, is unique as an entropy solution in the sense of Kruzhkov. These results establish in an indirect way the existence and uniqueness for bounded solutions of Problem (4.1) - (4.2). In addition they demonstrate some qualitative, characteristic properties of the solutions $v_{0}$. Below we shall discuss these properties and make a comparision with the numerical results.

(i) $v_{0}$ is symmetric in $\zeta$. That is

$$
v_{0}(\eta, \zeta)=v_{0}(\eta,-\zeta) \text { for all }(\eta, \zeta) \in \mathbb{R}^{2} .
$$

This follows directly from symmetry properties of equation (4.1) together with uniqueness.

(ii) There exists a constant $L>0$, such that

$$
v_{0}(\eta, \zeta)=0 \text { outside the strip } S=\{(\eta, \zeta): 0<\eta<L,-\infty<\zeta<\infty\} .
$$

To show this one needs to assume that $v_{0}$ decays to zero sufficiently fast as $|\eta|,|\zeta| \rightarrow \infty$. Then the argument is as follows. First write equation (4.1) in divergence form

$$
\frac{\partial}{\partial \eta}\left\{\frac{3-p}{2 p} \eta v_{0}-v_{0}^{\nu}\right\}+\frac{\partial}{\partial \zeta}\left\{\frac{1}{2} \zeta v_{0}+\frac{\partial v_{0}}{\partial \zeta}\right\}=0
$$

and introduce the transversal mass

$$
M_{t}(\eta)=\int_{-\infty}^{+\infty} v_{0}(\eta, \zeta) d \zeta \quad \text { for }-\infty<\eta<\infty .
$$

Integration of (4.5) witl respect to $\zeta$ yields

$$
\frac{3-p}{2 p} \frac{d}{d \eta}\left(\eta M_{t}\right)-\frac{d}{d \eta} \int_{-\infty}^{+\infty} v_{0}^{p}(\eta, \zeta) d \zeta=0
$$


and thus

$$
\frac{3-p}{2 p} \eta M_{t}-\int_{-\infty}^{+\infty} v_{0}^{p}(\eta, \zeta) d \zeta=C \quad \text { for }-\infty<\eta<\infty
$$

where $C$ is a constant of integration. Letting $|\eta| \rightarrow \infty$ in this expression and using the decay of $v_{0}$ at infinity yields $C=0$ and leaves us with

$$
\frac{3-p}{2 p} \eta M_{t}=\int_{-\infty}^{+\infty} v_{0}^{p}(\eta, \zeta) d \zeta \quad \text { for }-\infty<\eta<\infty
$$

Since $v_{0} \geq 0$ and consequently $M_{t} \geq 0$, this shows that $v_{0}(\eta, \zeta)=0$ for all $\eta \leq 0$.

From (4.9) we further obtain

$$
\frac{3-p}{2 p} \eta M_{t} \leq \sup _{\eta \in \mathbb{R}} v_{0}^{p-1}(\eta, \zeta) M_{\ell}
$$

or

$$
\left(\frac{3-p}{2 p} \eta-\sup _{\zeta \in \mathbb{R}} v_{0}^{p-1}(\eta, \zeta)\right) M_{\ell} \leq 0 .
$$

This implies the existence of a constant $L>0$, depending on the maximum value of $v_{0}$, such that $M_{t}(\eta)=0$ for all $\eta>L$. This establishes the second assertion.

Inequality (4.11) also implies

(iii)

$$
\sup _{\zeta \in \mathbb{R}} v_{0}^{p-1}(\eta, \zeta) \geq \frac{3-p}{2 p} \eta \quad \text { for } \quad 0 \leq \eta \leq L
$$

Having shown that the maximum value of $v_{0}^{p-1}$ with respect to $\zeta$ is strictly positive for each $0<\eta<L$ it follows that (Escobedo (1993b))

(iv)

$$
v_{0}>0 \text { in } S \text {. }
$$

It is also possible to estimate $v_{0}$ from above in $S$. To be specific we have

(v)

$$
v_{0}(\eta, \zeta) \leq\left(\frac{\eta}{p}\right)^{\frac{1}{p-1}} \text { for }(\eta, \zeta) \in S .
$$

Note that the upper bound is the one dimensional solution (3.12). Therefore this inequality has an obvious physical interpretation. The proof of (4.14) follows from a simple comparison argument.

The previous estimate implies $\partial v_{0}^{p-1} / \partial \eta(0, \zeta) \leq \frac{1}{p}$ for all $-\infty<\zeta<\infty$. The following assertion says that this inequality holds throughout $S$.

(vi)

$$
\frac{\partial v_{0}^{p-1}}{\partial \eta} \leq \frac{1}{p} \text { in } S .
$$

To prove this estimate one first writes the equation for $w=v_{0}^{p-1}$ and after that the equation for the derivative $z=\partial w / \partial \eta$. This latter equation has the constant solution $z=1 / p$. A comparision argument yields (4.15). 
Estimate (4.15) can be viewed as an entropy condition: a solution $v_{0}$ of (4.1) may admit a shock discontinuity in the $\eta$-direction only if (4.15) is not violated. This means that $v_{0}$ can only decrease across a shock. Note that (4.15), after integration, yields estimate (4.14).

The next result is concerned with the behaviour of $v_{0}$ as $|\zeta| \rightarrow \infty$. Let

$$
M_{L}(\zeta)=\int_{0}^{L} v_{0}(\eta, \zeta) d \eta
$$

denote the longitudinal mass. We have

(vii)

$$
M_{L}(\zeta)=\frac{M}{2 \sqrt{\pi}} e^{-\zeta^{2} / 4} \text { for all }-\infty<\zeta<\infty .
$$

To show this we integrate equation (4.5) with respect to $\eta$. Setting the constant of integration equal to zero we find the equation

$$
\frac{d M_{L}}{d \zeta}+\frac{1}{2} \zeta M_{L}=0
$$

and consequently

$$
M_{L}(\zeta)=M_{L}(0) e^{-\zeta^{2} / 4}, \quad-\infty<\zeta<\infty .
$$

The value for $M_{L}(0)$ follows from (4.2).

Combining (vi) and (vii) yields exponential decay of $v_{0}$ as $|\zeta| \rightarrow \infty$. We give the result without proof.

(viii)

$$
v_{0}^{p}(\eta, \zeta) \leq \frac{M(p-1)}{\sqrt{\pi} p} e^{-\zeta^{2} / 4} \text { for all }(\eta, \zeta) \in S .
$$

This inequality allows us to estimate the magnitude of $L$. Using (4.12) we find

$$
L \leq \frac{2 p}{3-p}\left(\frac{M(p-1)}{\sqrt{\pi} p}\right)^{\frac{p-1}{p}} .
$$

We conclude the list of properties with two conjectures about the behaviour of $v_{0}$ near $\eta=0$ and $\eta=L$. These conjectures will have the form of asymptotic expansions and will be supported later on by the numerical results.

We start off with the behaviour near $\eta=0$. As a first observation we note that equation (4.1) has a separable solution which vanishes at $\eta=0$ and for large $|\xi|$. It is given by

$$
v(\eta, \zeta)=\left(\frac{p-1}{p} \eta\right)^{\frac{1}{p-1}} f_{0}(\zeta) \text { for } \eta>0 \text { and }-\infty<\zeta<\infty,
$$

where the function $f_{0}$ satisfies the ordinary differential equation

$$
f_{0}^{\prime \prime}+\frac{\zeta}{2} f_{0}^{\prime}+\frac{f_{0}}{p-1}-f_{0}^{p}=0,-\infty<\zeta<\infty
$$


and the boundary conditions

$$
f_{0}( \pm \infty)=0 .
$$

In (4.23) the primes denote differentiation with respect to $\zeta$. The boundary value problem (4.23), (4.24) was studied by Brezis et al. (1986). They proved the existence of a solution $f_{0}$ satisfying $\max _{\zeta \in \mathbb{R}} f_{0}(\zeta)=f_{0}(0)<\left(\frac{1}{p-1}\right)^{\frac{1}{p-1}}, \zeta f^{\prime}(\zeta)<0$ for $\zeta \neq 0$ and $f_{0}(\zeta)$ decays to zero exponentially as $|\zeta| \rightarrow \infty$. The ansatz about the behaviour of $v_{0}$ near $\eta=0$ is the following:

(ix) For each $\zeta \in \mathbb{R}$,

$$
\frac{v_{0}(\eta, \zeta)}{\left(\frac{p-1}{p} \eta\right)^{\frac{1}{p-1}}}-f_{0}(\zeta)=0\left(\eta^{\lambda}\right) \text { as } \eta \downarrow 0,
$$

where $\lambda$ is a positive constant. It appears as the eigenvalue of a linear problem which is given in the appendix.

The behaviour of $v_{0}$ as $\eta \uparrow L$ is more complicated. We conjecture that for any $\zeta \neq 0$,

$$
\lim _{\eta \mid L} v_{0}(\eta, \zeta)=0
$$

while at $\zeta=0$

$$
\lim _{\eta \mid L} v_{0}(\eta, 0)=v_{0}\left(L^{-}, 0\right)>0 .
$$

Thus $v_{0}$ has a nose-like profile at $(L, 0)$, in which a singularity at $\eta=L$ and $\zeta=0$ creates a transverse flow of particles. In the appendix we derive the behaviour of $v_{0}$ near $(L, 0)$, using the method of matched asymptotic expansions. It gives

$$
v_{0}\left(L^{-}, 0\right)=\left(\frac{3-p}{2 p} L\right)^{\frac{1}{p-1}}
$$

indicating that inequality (4.12) is sharp at the singularity. The structure of the solution near the nose is shown in Figure A1 of the appendix. Sufficiently close to $\eta=L$ two transition layers, taking $v_{0}$ from $v_{0}=0$ to $v_{0}=\left(\frac{3-p}{2 p} L\right)^{1 / p-1}$, move towards each other as $\eta \uparrow L$, eventually coalescing at $\eta=L$ to produce the singularity at the nose. Thus the solution approaches this singularity in a plateau like fashion. A feature of this structure is the locus of the centre of the transition layer as $\eta \uparrow L$. In terms of $(\zeta, \eta)$ the locus is given by expression (A.18), having the form

$$
|\zeta|=2 \sqrt{\frac{p}{(p-1)(3-p)}}\left\{\frac{(L-\eta)}{L} \log \left(\frac{L}{L-\eta}\right)\right\}^{1 / 2} .
$$

Now we compare the analytical results with the numerical solution. To compute a solution of Problem (4.1), (4.2) directly, using a finite difference or finite element method say, is fraught with difficulties. Therefore we used the computed large time solution of 
Problem (IVP), with the appropriate scalings, as a comparison for the analytical properties. In Figure 4.1, we show the scaled large time solution of Problem $(I V P)$ for $p=1.2$ and $u_{0}$ as in Figure 2.1, with $M=8$.

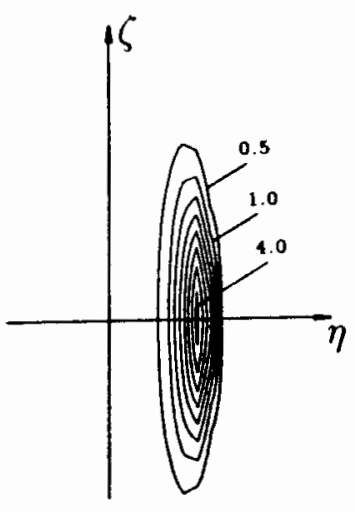

(a)

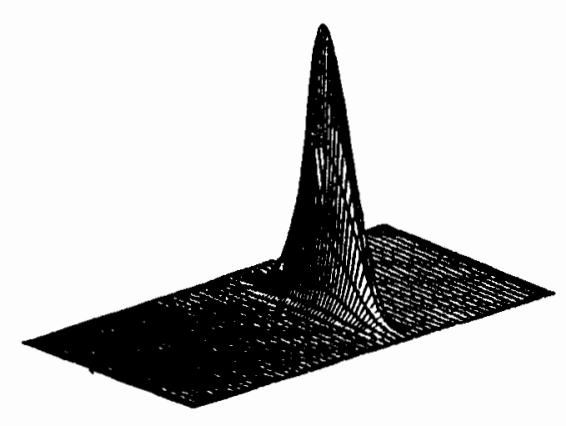

(b)

Fig. 4.1: Numerical solution of Problem (IVP) in the scaled variables $v=t^{\frac{3}{2 p}} u$ and $\eta=(t-x) / t^{\frac{3-p}{2 p}}, \zeta=y / t^{1 / 2}$. IIere $p=1.2, M=8$ (as in Figure 2.1) and $t=4500$. In (a) the level curves of the solution are shown, while (b) shows the solution as a three dimensional object.

These numerical results clearly confirm the large time scalings of Section 2 and also our analytic findings set out above. In particular the asymptotic behaviour near the singular point $(L, 0)$ is certainly validated by the computations.

\subsection{The reduced equation for $0<p<1$}

Here the problem is to find a function $v_{0}=u_{0}^{m}$, with $m=\frac{1}{p}$, such that (see equation (2.32))

$$
\frac{3}{3 m-1} u_{0}+\frac{2}{3 m-1} \eta \frac{\partial u_{0}}{\partial \eta}+\frac{1}{3 m-1} \zeta \frac{\partial u_{0}}{\partial \zeta}-\frac{\partial u_{0}^{m}}{\partial \eta}+\frac{\partial^{2} u_{0}^{m}}{\partial \zeta^{2}}=0
$$

for all $(\eta, \zeta) \in \mathbb{R}^{2}$, and

$$
u_{0} \geq 0, \iint_{\mathbb{R}^{2}} u_{0} d \eta d \zeta=M
$$

Equation (4.27) too is second order in $\zeta$ but only first order in $\eta$. In addition there is the complication that the term describing $\zeta$-diffusion is degenerate since the corresponding diffusion coefficient $m u_{0}^{m-1}(m>1)$ vanishes as $u_{0} \searrow 0$. Because of this we expect less $\zeta$-smoothness than in the previous case. In fact, based on the observations made in the introduction about the finite speed of expansion of the region where $u>0$, we conjecture 
that there exist functions $l^{ \pm}(\eta),-\infty<\eta<\infty$, satisfying $l^{+} \geq 0$ and $l^{+}+l^{-}=0$ by symmetry, such that

$$
u_{0}(\eta, \zeta)=0 \text { for all }(\eta, \zeta) \in \mathbb{R}^{2} \text { with }|\zeta| \geq l^{+}(\eta)
$$

This behaviour is confirmed by the computalions. We note that to the authors knowledge equation (4.27) is new and no existence and uniqueness theory is yet available. In this respect our discussion on the behaviour of $u_{0}$ (or $v_{0}$ ) is purely formal. Again we compose a list of properties.

As mentioned above we expect

(i) $u_{0}$ is symmetric in $\zeta$.

Writing (4.27) in the divergence form

$$
\frac{\partial}{\partial \eta}\left\{\frac{2}{3 m-1} \eta u_{0}-u_{0}^{m}\right\}+\frac{\partial}{\partial \zeta}\left\{\frac{m}{3 m-1} \zeta u_{0}+\frac{\partial u_{0}^{m}}{\partial \zeta}\right\}=0
$$

and integrating this equation in $\zeta$, leads to the invariance property

$$
\frac{2}{3 m-1} \eta \int_{-\infty}^{+\infty} u_{0}(\eta, \zeta) d \zeta-\int_{-\infty}^{+\infty} u_{0}^{m}(\eta, \zeta) d \zeta=0
$$

for all $-\infty<\eta<\infty$. From this expression it follows that

(ii) There exists a constant $L>0$, depending on the maximum of $u_{0}$, such that

$$
u_{0}(\eta, \zeta)=0 \text { outside the strip }\{(\eta, \zeta): 0<\eta<L,-\infty<\zeta<\infty\}
$$

and also

(iii)

$$
\sup _{\zeta \in \mathbb{R}} u_{0}^{m-1}(\eta, \zeta)=\sup _{\zeta \in \mathbb{R}} v_{0}^{1-p} \geq \frac{2 p}{3-p} \eta
$$

for $0<\eta<L$.

For the support of $v_{0}$, that is the set where $v_{0}>0$, we expect a set as in Figure 4.2 below.

Indeed, when considering the asymptotic expansions near $\eta=0$ and $\eta=L$, we obtain strong evidence that $l^{+}(\eta) \searrow 0$ as $\eta \downarrow 0$ and as $\eta \uparrow L$, just as in Figure 4.2. The set where $v_{0}>0$ will be denoted by $S$.

Again we can make a comparison from above with the one dimensional solution. The result is

(iv)

$$
u_{0}(\eta, \zeta) \leq\left(\frac{\eta}{m}\right)^{\frac{1}{m-1}} \text { or } v_{0}(\eta, \zeta) \leq(p \eta)^{\frac{1}{1-p}} \text { for }(\eta, \zeta) \in S .
$$

This estimate implies

$$
\frac{\partial v_{0}^{1-p}}{\partial \eta}(0,0) \leq p
$$




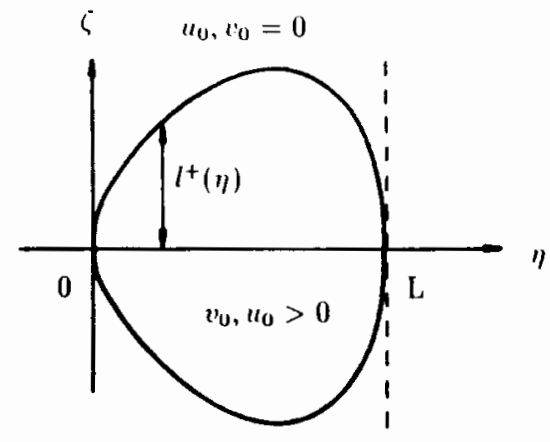

Fig. 4.2: Location of the support $(S)$ of $v_{0}$.

In fact, by the method described in the previous section, we can show the "entropy" inequality

(v)

$$
\frac{\partial u_{0}^{m-1}}{\partial \eta}=\frac{\partial v_{0}^{1-p}}{\partial \eta}(\eta, \zeta) \leq p \text { for all }(\eta, \zeta) \in S .
$$

It is possible to construct bounds on the solution which are in the spirit of identity (4.17) and estimate (4.20). However due to the different character of equation (4.27), such bounds are not as elegant as the previous ones and of a complicated technical nature. Therefore they will not be given here.

Next we turn to the asymptotic behaviour of the solution as $\eta \downarrow 0$ and as $\eta \uparrow L$. In the appendix we use formal asymptotic methods to show that, in terms of $v_{0}$,

(vi)

$$
v_{0}=(p \eta)^{1 / 1-p}\left[\left\{1-\frac{3(1-p) \zeta^{2}}{4 p(3-p) \eta|\log \eta|}\right\}^{1 / 1-p}+o(1)\right]
$$

as $\eta \downarrow 0$. This gives an emerging profile with the interface such that

$$
l^{ \pm}(\eta) \sim \pm \sqrt{\frac{4 p(3-p)}{3(1-p)}}\{\eta|\log \eta|\}^{1 / 2}
$$

as $\eta \downarrow 0$. This of course is entirely consistent with the finite nature of the support of $v_{0}$.

As $\eta \uparrow L$ we have a nose-type singularity very similar to that in the case $1<p<3 / 2$, except that the support of the solution is now finite (see also equation (A.2S)). Again two transition layers converge as $\eta \uparrow L$ coalescing to the singularity at $(L, 0)$ where 
(vii)

$$
v_{0}\left(L^{-}, 0\right)=\left(\frac{2 p L}{3-p}\right)^{1 / 1-p}
$$

which since $p<1$ is consistent with the bounds in (4.33) and (4.34). The locus of the transition layer centres is given by expression (A.20) as

$$
|\zeta|=\sqrt{\frac{2}{1-p}}\left\{(L-\eta) \log (L /(L-\eta)\}^{1 / 2}\right.
$$

which, except for the scaling factor, is equivalent to the corresponding form for $p>1$.

As in the case $1<p<3 / 2$ we compare our analytic results with the large time solutions of the full problem (IVP) since the task of computing numerical solutions of the reduced equation directly again poses considerable difficulties. We present these solutions in Figure 4.3. These are clearly consistent with the large time scalings of Section 2 and the analytic properties presented above including the asymptotic results encapsulated in (4.38) and (4.40).

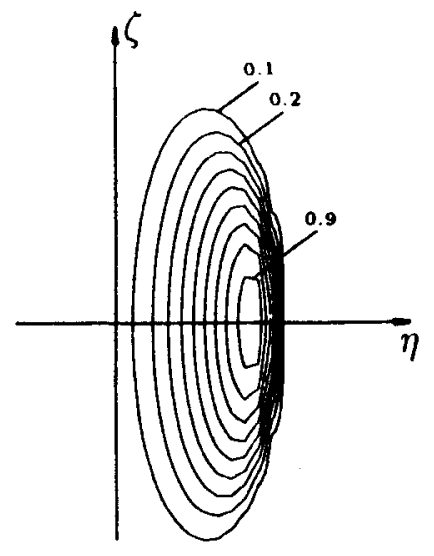

(a)

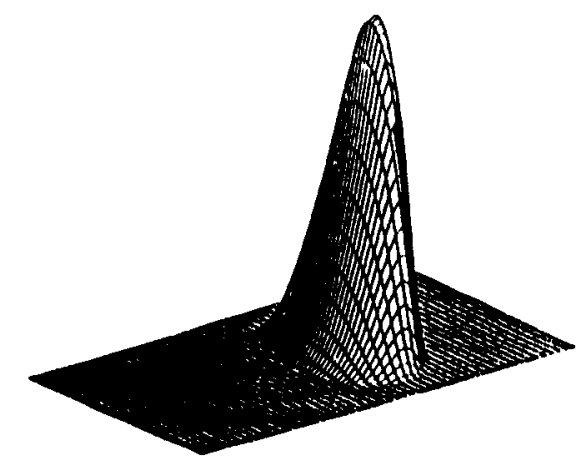

(b)

Fig. 4.3: Numerical solution of Problem (IVP) in the scaled variables $v=t^{3 /(3-p)} u$ and $\eta=x t^{-2 p /(3-p)}, \zeta=y t^{-p /(3-p)}$, with $p=0.5$ and $M=S$. In (a) the level curves for the solution with $t=3000$ are shown, while for the same value of $t$ (b) shows the solution as a three dimensional object.

\section{Development of contaminant plumes}

When considering the groundwater transport of a contaminant plume at field scale from a practical point of view, one is often only interested in certain averaged quantities (moments) such as the mean displacement or the transversal and lateral spreading. The reason for this 
is that small scale variations in the physical and chemical properties of the porous medium disturb the actual contaminant distribution. For instance it is now well accepted that spatial variations in water velocity, caused in turn by the heterogeneity of the hydraulic conductivity, can be accounted for by a macro-dispersion at field scale $\left(D_{x x}\right.$ and $D_{y y}$ in equation (1.1)).

The effect of chemical heterogeneity for instance, through a spatial variation of the coefficient $K$ in (1.2), was investigated only recently. Dagan $\&$ Cvetlovic (1994) and Burr $\&$ Sudicky (1994) considered the linear case $(p=1)$, while Bosma et al. (1994) considered the Freundlich case $(0<p<1)$. Bosma et al. used a Monte Carlo method in a medium in which the hydraulic conductively and the Freundlich coeflicient $K$ were $\log$ normal distributed with a certain degree of correlation. They showed that the results obtained in this paper can also be applied to transport problems in heterogeneous media, if one makes a comparison based on moments and in particular if one compares the time evolution of the moments.

The relevant moments are:

(i) dissolved mass

$$
M(t)=\iint_{\mathbb{R}^{2}} C(x, y) d x d y
$$

(ii) centre of mass in mean flow direction

$$
\eta_{\mathbf{x}}(t)=\frac{1}{M(t)} \iint_{\mathbf{R}^{2}} x C(x, y) d x d y
$$

(iii) longitudinal variance

$$
\tau_{x x}^{2}(t)=\frac{1}{M(t)} \iint_{\mathbb{R}^{2}}\left(x-\eta_{x}(t)\right)^{2} C(x, y) d x d y
$$

(iv) transversal variance

$$
\tau_{y y}^{2}(t)=\frac{1}{M(t)} \iint_{\mathbf{R}^{2}} y^{2} C(x, y) d x d y
$$

Substituting the asymptotic forms obtained in section 2 into these expressions, yields their large time behaviour. Moreover, by applying the scaling (1.5) or (1.6) and by absorbing the total mass

$$
M_{C}:=\iint_{\mathbf{R}^{2}}\left\{0 C_{0}+R_{R} K C_{0}^{p}\right\} d x d y
$$

into an additional scaling, we obtain explicity the dependence of the large time behaviour of the moments on the relevant physical parameters. For instance, if $0<p<1$ we find as $t \rightarrow \infty$ that

$$
\eta_{x}(t)=\left(\frac{M_{C}}{\rho K}\right)^{\frac{2(1-p)}{3-p}}\left(\frac{v}{D_{y y}}\right)^{\frac{1-p}{3-p}}\left(\frac{0 v t}{\rho K}\right)^{\frac{2 p}{3-p}} \mu(p)\{1+o(1)\}
$$


where

$$
\begin{gathered}
\mu(p):=\frac{\iint_{\mathbb{R}^{2}} \eta \tilde{v}_{0}(\eta, \zeta) d \eta d \zeta}{\iint_{\mathbb{R}^{2}} \tilde{v}_{0}(\eta, \zeta) d \eta d \zeta} \\
\tau_{x x}(z)=\left(\frac{M_{C}}{\rho K}\right)^{\frac{4(1-p)}{3-p}}\left(\frac{v}{D_{y y}}\right)^{\frac{2(1-p)}{3-p}}\left(\frac{0 v t}{\rho K}\right)^{\frac{4 p}{3-p}} \tau_{L}^{2}(p)\{1+o(1)\}
\end{gathered}
$$

where

$$
\tau_{L}^{2}(p):=\frac{\iint_{\mathbb{R}^{2}}(\eta-\mu(p))^{2} \tilde{v}_{0}(\eta, \zeta) d \eta d \zeta}{\iint_{\mathbb{R}^{2}} \tilde{v}_{0}(\eta, \zeta) d \eta d \zeta} ;
$$

and

$$
\tau_{y y}(z)=\left(\frac{M_{C}}{\rho K}\right)^{\frac{2(1-p)}{3-p}}\left(\frac{v}{D_{y y}}\right)^{\frac{2}{2-p}}\left(\frac{0 v t}{\rho K}\right)^{\frac{2 p}{3-p}} \tau_{T}^{2}(p)\{1+o(1)\},
$$

where

$$
\tau_{T}^{2}(p):=\frac{\iint_{\mathbb{R}^{2}} \zeta^{2} \tilde{v}_{0}(\eta, \zeta) d \eta d \zeta}{\iint_{\mathbb{R}^{2}} \dot{v}_{0}(\eta, \zeta) d \eta d \zeta} .
$$

The function $\tilde{v}_{0}$ appearing in the coefficients $\mu(p), \tau_{L}^{2}(p)$ and $\tau_{T}^{2}(p)$ is the solution of (2.32), (2.33) with $M=1$. Their value has to be established numerically which we did for $p=1 / 2$ to give

$$
\mu\left(\frac{1}{2}\right)=0.656, \tau_{L}^{2}\left(\frac{1}{2}\right)=0.052 \text { and } \tau_{T}^{2}\left(\frac{1}{2}\right)=0.853 .
$$

Expression (5.6) - (5.11) we used in the paper by Bosma et al. to interpret the movement and spreading of a contaminant plume in a heterogeneous medium. In many cases the agreement between their Monte Carlo simulation for the heterogeneous medium and our analytical expression were excellent, in particular with respect to the exponent of $t$.

Finally we make an observation concerning the motion of the $y$ coordinate of the centre of mass, namely

$$
\mu_{y}(t)=\frac{1}{M(t)} \iint_{\mathbb{R}^{2}} y C(x, y, t) d x d y .
$$

If we use the leading order term from expansion (2.2) $m(5.13)$ we find that $\mu_{y}(t)=0$ identically, due to the symmetry of $V_{0}$ and an function of $\zeta$. The large time behaviour of $\mu_{y}(t)$ must therefore be determined by the non-symmetric higher order terms in the expansion of $c(x, y, t)$ for $t$ large. These reflect, in some unspecified way, the asymmetric nature of the initial data.

We conclude this section with a short description of the algorithm used to generate the two-dimensional numerical results of this paper. It is based on the "conservative form" of the differential equation (1.7), obtained by first transforming to a moving coordinate system as in (1.10) and making the change of variables $s=u+u^{p}, \phi(s)=u$. The result is a nonlinear convection diffusion equation in $s$. The convection terms are handled numerically using a higher-order Godunov scheme described in Bell et al. (1988). These terms are incorporated explicitly in time. Diffusion is handled using an implicit, cell-centered finite difference method. The combination of higher-order Godunov methods with cell-centered 
finite differences for multidimensional convection-diffusion equations was formulated and analysed in Dawson (1993a). Analysis for the nonlinear contaminant transport problem posed here is given in Dawson (1993b).

In the simulation presented here, the computational domain and the computational mesh varied depending on the exponent $p$. In all cases, the domain was chosen large enough so that boundaries did not effect the numerical solution. A typical computation used 160,000 uniform grid blocks on a computational domain $[-200,200] \times[-200,200]$. However, in some cases, most notably $p=1.2$, the computational domain was much larger (due to the long times simulated) and a nonuniform mesh was used. At various times during the simulation the domain was regridded and the numerical solution was projected conservatively onto the new mesh. For stability of the advection scheme, the time-step in all simulations satisfied a CFL constraint.

We attempted various approaches for solving (4.1) - (4.2) numerically with little success. This equation represents a nonlinear, stationary convection equation for $v_{0}$ in the $\eta-\zeta$ plane. As one approach, we added an artificial diffusion term to stabilize the equation and applied an upwinded finite difference method, treating the nonlinearities using Picard iteration. The convergence of this iterative procedure was very slow, with the number of iterations exceeding the number of time-steps needed to drive the original equation (Problem IVP) to steady-state. We concluded that simulating the time-dependent equation until steady-state was reached gave more reliable answers with less computational effort.

\section{Appendix}

Local solutions of the reduced equations for $p<3 / 2$ near the tail and nose of the limiting profile

In this appendix we contruct local solutions of the reduced equations (2.22) and (2.32) near $\eta=0$ and $\eta=L$.

\section{A.1 $1<p<3 / 2$}

Here we take equation (2.22) and put

$$
v_{0}=\left\{\frac{\eta(p-1)}{p}\right\}^{1 / p-1} w_{0}\left(\eta_{1}, \zeta\right)
$$

where $\eta_{1}=\eta / L$ to give

$$
\frac{\partial^{2} \omega_{0}}{\partial \zeta^{2}}+\frac{\zeta}{2} \frac{\partial \omega_{0}}{\partial \zeta}+\frac{\omega_{0}}{(p-1)}-\omega_{0}^{p}=-\frac{(3-p)}{2 p}\left\{1-\frac{2 p(p-1)}{(3-p)} \omega_{0}^{p-1}\right\} \eta_{1} \frac{\partial \omega_{0}}{\partial \eta_{1}}
$$

Near the tail of the profile located at $\eta_{1}=0$ we seek a local solution of the form

$$
\omega_{0}\left(\eta_{1}, \zeta\right)=f_{0}(\zeta)+\eta_{1}^{\lambda} f_{1}(\zeta)+\cdots
$$


Clearly the leading order term $f_{0}(\zeta)$ satisfies

$$
f_{0}^{\prime \prime}+\frac{\zeta}{2} f_{0}^{\prime}+\frac{f_{0}}{(p-1)}-f_{0}^{p}=0
$$

together with the boundary conditions

$$
f_{0}( \pm \infty)=0 .
$$

We note here that $f_{0}(\zeta)$ is actually an exact solution of (A.2) and has been studied in a different context by Brezis, Peletier \& Terman (1986). In that paper they showed that there exists a unique symmetric solution of (A.4) satisfying (A.5) with exponential decay as $|\zeta| \rightarrow \infty$. These are the very properties we require here. We observe that $f_{0}$ also satisfies the integral condition

$$
\int_{-\infty}^{\infty}\left[\frac{(3-p) f_{0}}{2(p-1)}-f_{0}^{p}\right] d \zeta=0
$$

which is related to condition (4.9).

Going further in the expansion (A.3) we find that $f_{1}(\zeta)$ has to satisfy

$$
\begin{gathered}
f_{1}^{\prime \prime}+\frac{\zeta}{2} f_{1}^{\prime}+f_{1}\left\{\frac{1}{(p-1)}+\frac{(3-p) \lambda}{2 p}-f_{0}^{p-1}[p+(p-1) \lambda]\right\}=0 \\
f_{1}( \pm \infty)=0 .
\end{gathered}
$$

This is an eigenvalue problem for $f_{1}(\zeta)$ with a point spectrum for real $\lambda$ (see example Titchmarsh (1946)).

We now turn to the behaviour at the nose of the profile near $\eta_{1}=1$. Here we make the local scaling

$$
\xi=\zeta / \rho^{1 / 2}, \rho=1-\eta_{1}
$$

in (A.2) to give

$$
\begin{gathered}
\frac{\partial^{2} \omega_{0}}{\partial \xi^{2}}+\rho\left\{\frac{\xi}{2} \frac{\partial \omega_{0}}{\partial \xi}+\frac{\omega_{0}}{(p-1)}-\omega_{0}^{p}\right\} \\
=(1-\rho) \frac{(3-p)}{2 p}\left\{1-\frac{2 p(p-1)}{(3-p)} \omega_{0}^{p-1}\right\}\left\{\rho \frac{\partial \omega_{0}}{\partial \rho}-\frac{\xi}{2} \frac{\partial \omega_{0}}{\partial \xi}\right\} .
\end{gathered}
$$

We now make the expansion

$$
\omega_{0}(\rho, \xi)=\left[\frac{(3-p)}{2(p-1)}\right]^{1 / p-1}+\rho^{1 / 2} W_{1}(\xi)+0(\rho)
$$

for $\rho \rightarrow 0, \xi=0(1)$. Substituting (A.9) into (A.S) and equating terms which are $0\left(\rho^{1 / 2}\right)$ gives

$$
W_{1}^{\prime \prime}-\frac{1}{2} \xi_{1} W_{1}^{\prime}+\frac{W_{1}}{2}=0
$$


where

$$
\xi_{1}=\sqrt{\frac{(p-1)(3-p)}{2 p}} \xi
$$

and primes denote differentration with respect to $\xi_{1}$. The even solution of this equation is given by

$$
W_{1}\left(\xi_{1}\right)=A-A \xi_{1} \int_{0}^{\xi_{1}}\left\{e^{s^{2} / 4}-1\right\} \frac{d s}{s^{2}}
$$

where $A=W_{1}(0)$ is an arbitrary positive constant. Anticipating a possible nonuniformity in (A.9), we look at the behaviour of the expansion (A.9) as $\left|\xi_{1}\right| \rightarrow \infty$. Now from (A.11) we have for $\left|\xi_{1}\right| \rightarrow \infty$

$$
W_{1}\left(\xi_{1}\right) \sim-\frac{2 A}{\xi_{1}^{2}} e^{\xi_{1}^{2} / 4}
$$

so the first two terms in (A.9) give

$$
\omega_{0}\left(\rho, \xi_{1}\right) \sim\left\{\frac{(3-p)}{2(p-1)}\right\}^{1 / p-1}-\frac{2 \rho^{1 / 2} A e_{1}^{\xi_{1}^{2} / 4}}{\xi_{1}^{2}}+\cdots
$$

which reveals a nonuniformity where

$$
\xi_{1}^{2} \sim \rho^{1 / 2} e^{\xi_{1}^{2} / 4}
$$

It is interesting to point out here that a similarly structured nonuniformly arises in Grundy $\&$ Peletier (1987). We observe that (A.12) defines a variable $\chi$ where, for $\xi_{1}>0$, (there is an equivalent transition region for $\left.\xi_{1}<0\right)$,

$$
\begin{gathered}
\xi_{1}=\sqrt{2}\{\log (1 / \rho)\}^{1 / 2}+\frac{\sqrt{2} \log \{\log (1 / \rho)\}}{[\log (1 / \rho)]^{1 / 2}} \\
+\frac{\sqrt{2} \chi}{[\log (1 / \rho)]^{1 / 2}}+\cdots
\end{gathered}
$$

such that $\chi=0(1)$ when $(A .12)$ is satisfied. We now rewrite $(A .8)$ in terms of $\chi$ and $\rho$ and expand $\omega_{0}(\chi, \rho)$ in the form

$$
\omega_{0}(\chi, \rho)=Z_{0}(\chi)+\frac{Z_{1}(\chi)}{\log (1 / \rho)}+\cdots
$$

and find that $Z_{0}(\chi)$ satisfies

$$
Z_{0}^{\prime \prime}=-\frac{\left\{1-\frac{2 p(p-1)}{(3-p)} Z_{0}^{p-1}\right\}}{(p-1)} Z_{0}^{\prime}
$$

Now for $\xi_{1}>0$ the solution of (A.15) has to talie $Z_{0}$ from the plateau value $\left\{\frac{3-p}{2(p-1)}\right\}^{1 / p-1}$ as $\chi \rightarrow-\infty$ to zero as $\chi \rightarrow+\infty$. The solution which does this is given by

$$
Z_{0}=\left\{\frac{(3-p)}{2(p-1)\left\{h e^{x}+1\right\}}\right\}^{1 / p-1}
$$


where the constant $K$ can be found in terms of $A$ by matching the expansions (A.14) and (A.9). This gives

$$
K=(p-1) A\left\{\frac{2(p-1)}{3-p}\right\}^{1 / p-1}
$$

and $A$ is not determined by the local analysis.

To summarise we have that as $\eta_{\mathrm{l}} \uparrow 1$

(a) $\xi=\frac{s}{\left(1-\eta_{1}\right)^{1 / 2}}=0(1)$

$$
\omega_{0}=\left\{\frac{3-p}{2(p-1)}\right\}^{1 / p-1}+\left(1-\eta_{1}\right)^{1 / 2} W_{1}\left\{\zeta /\left(1-\eta_{1}\right)^{1 / 2}\right\}+o\left(1-\eta_{1}\right)^{1 / 2}
$$

(b) $x=0(1)$

$$
\omega_{0}=\left\{\frac{(3-p)}{2(p-1)\left\{K e^{x}+1\right\}}\right\}^{1 / p-1}+0(1)
$$

a structure which is shown schematically in Figure A.1.

The locus of the centre of the transition region is given by putting $x=0$ in (A.13). To leading order this gives in the original coordinates

$$
\zeta= \pm 2 \sqrt{\frac{p}{(p-1)(3-p)}}\left\{\frac{(L-\eta)}{L} \log \left(\frac{L}{L-\eta}\right)\right\}^{1 / 2}
$$

as $\eta \uparrow L$.

This behaviour is confirmed by the numerical results shown in Section 4, see Figure 4.1.

A.2. $0<p<1$

For this range of values of $p$ we start from equation (2.32)

$$
\frac{\partial^{2} v_{0}}{\partial \zeta^{2}}-\frac{\partial v_{0}}{\partial \eta}+\frac{p}{(3-p)} \zeta \frac{\partial\left(v_{0}^{p}\right)}{\partial \zeta}+\frac{2 p}{(3-p)} \eta \frac{\partial\left(v_{0}^{p}\right)}{\partial \eta}+\frac{3 p}{(3-p)} v_{0}^{p}=0
$$

where $0<\eta<L$. To simplify matters we put

$$
v_{0}=\{p \eta\}^{1 / 1-p} \omega_{0}(r, \eta)
$$

where

$$
r=\zeta / \eta^{1 / 2}
$$

This gives the equation

$$
\frac{\partial^{2} \omega_{0}}{\partial r^{2}}+\frac{r}{2} \frac{\partial \omega_{0}}{\partial r}+\frac{\left(\omega_{0}^{p}-\omega_{0}\right)}{(1-p)}=\eta \frac{\partial}{\partial \eta}\left\{\omega_{0}-\frac{2 \omega_{0}^{p}}{(3-p)}\right\}
$$




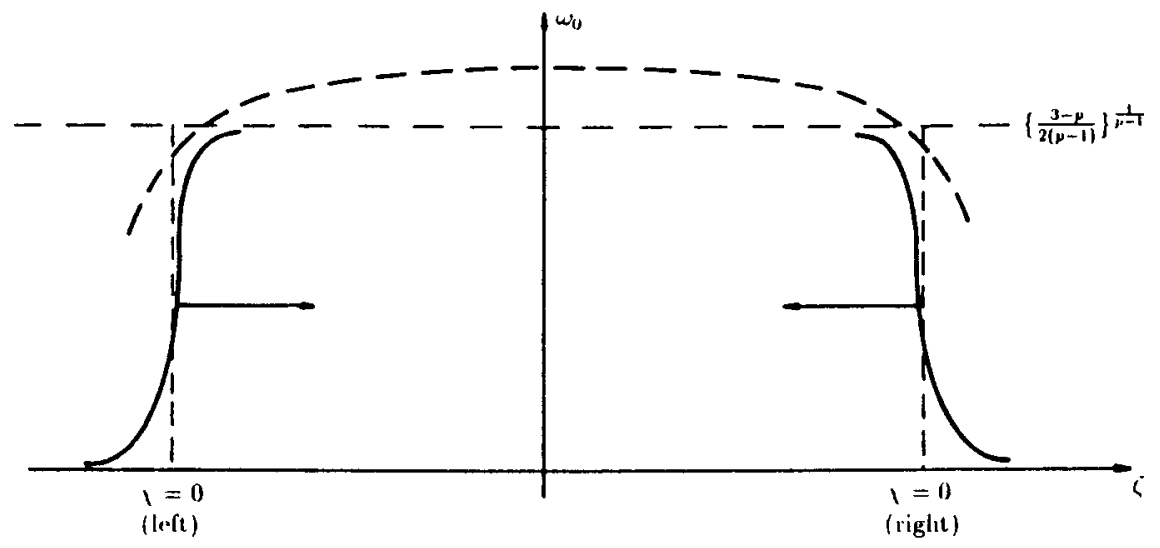

Fig. A1 Plateau like structure of the local solution near $\eta=L$. The two transition layers move towards each other as $\eta \uparrow L$ and coalesce at $\eta=L$. The two term solution (A.9) is indicated by the broken line and the two transition solutions, given by $(A .16)$ and its counterport for $\zeta<0$, by the solid lines. The locus of the centre of the transition region $(\chi=0 \mathrm{right} /$ left $)$ is given by (A.18) to leading order as $\eta \uparrow L$.

We are interested in the way the profile developes as $\eta$ increases from zero at the tail. If we try to construct the equivalent to (A.3) for $p>1$, we find that the only bounded solution for $\omega_{0}(r)$ is $\omega_{0}=1$, a solution which does not satisfy the boundary conditions at the edge of the support. The situation is very similar to that for the extinction problem for diffusion reaction equations (Grundy (1992)) and with this analogy in mind we seek an expansion for $\omega_{0}(r, \eta)$ as $\eta \rightarrow 0$ of the form

$$
\omega_{0}=1+\frac{V_{1}(r)}{\log (\eta)}+\frac{V_{2}(r) \log (\log \eta)}{(\log \eta)^{2}}+\frac{V_{3}(r)}{(\log \eta)^{2}}+\cdots
$$

Substituting (A.21) into (A.20) we find that $V_{1}$ satisfies

$$
V_{1}^{\prime \prime}+\frac{r V_{1}^{\prime}}{2}-V_{1}=0
$$

and, disregarding $V_{2}$, that $V_{3}(r)$ satisfies

$$
V_{3}^{\prime \prime}+\frac{r V_{3}^{\prime}}{2}-V_{3}=\frac{p}{2} V_{1}^{2}-\frac{3(1-p)}{(3-p)} V_{1}
$$

We need polynomial solutions of these equations so we take

$$
V_{1}=A\left(2+\zeta^{2}\right)
$$


where $A$ is found by requiring that the equation (A.23) for $V_{3}$ has a polynomial particular integral. This gives

$$
A=\frac{-3(1-p)}{4 p(3-p)}
$$

Clearly, the form (A.24) for $V_{1}$ implies that the ordering of expansion (A.21) breaks down where the new variable

$$
s=r /|\log \eta|^{1 / 2}=0(1)
$$

with a corresponding region of nonuniformity when $s<0$. Introducing the variable $s$ into (A.20) together with the expansion

$$
\omega_{0}=U_{0}(s)+o(1)
$$

as $\eta \rightarrow 0, s=0(1)$, we find that the resulting equation for $U_{0}$ can be solved to yield

$$
U_{0}(s)=\left\{1-\frac{3(1-p) s^{2}}{4 p(3-p)}\right\}^{1 / 1-p} .
$$

Reverting to the original variables we find that

$$
\omega_{0} \sim\left\{1-\frac{3(1-p) \zeta^{2}}{4 p(3-p) \eta|\log \eta|}\right\}^{1 / 1-p}
$$

as $\eta \rightarrow 0$, a profile that has an interface where

$$
\zeta^{2}=\frac{4 p(3-p)}{3(1-p)} \eta|\log \eta|
$$

We finally note that the equivalent invariance condition to (A.6) for $1<p<3 / 2$ is

$$
\int_{-\infty}^{\infty}\left\{U_{0}-\frac{2 U_{0}^{p}}{(3-p)}\right\} d s=0
$$

which, using (A.24), can be written as

$$
\int_{-1}^{1}\left(1-t^{2}\right)^{p / 1-p}\left(\frac{1-p}{3-p}-t^{2}\right) d t=0
$$

which can be easily verified.

The structure of the profile near the nose for $0<p<1$ is almost identical to that for $1<p<3 / 2$ except that the support is finite in $\zeta$. Starting from (A.20) we have for $\eta \uparrow L$ the results

(a) $t=\frac{r}{(L-\eta)^{1 / 2}}=0(1)$

$$
\omega_{0}=\left(\frac{2}{3-p}\right)^{1 / 1-p}+(L-\eta)^{1 / 2} W_{1}\left\{r /(L-\eta)^{1 / 2}\right\}+o(L-\eta)^{1 / 2}
$$


(b) $\chi=0(1)$

$$
\omega_{0}=\left\{\frac{2}{(3-p)}-C e^{x}\right\}^{1 / 1-p}+o(1)
$$

where matching gives $C(A)$ and where $A$ is a constant appearing in the expression for $W_{1}$. Note that $\omega_{0}$ vanishes at a finite value of $\chi$ which is consistent with the finite nature of the support of $v_{0}$. The centre of the transition region, $\chi=0$, is given to leading order as $\eta \uparrow L$ by

$$
\zeta= \pm \sqrt{\frac{2}{1-p}}\{(L-\eta) \log (L / L-\eta)\}^{1 / 2},
$$

Again these results compare well with the numerical solution given in Section 4, see Figure 4.3.

The schematical structure of the local solution is similar to that exhibited in Figure A.1. except of course that the support is finite in $\zeta$.

Acknowledgement The authors would like to thank the British Council-NWO for financial support during the course of this work.

\section{References}

[1] J. Aguirre, M. Escobedo and E. Zunzun, Self-similar solutions of a convection diffusion equation and related semilinear elliptic problems, Comm. in Partial Differential Equations, 15, 139-157, 1990.

[2] D.G. Aronson, The Porous Media Equation, in Nonlinear Diffusion Problems, Lecture Notes in Mathematics, No. 1224, (Edited by A. Fasano and M. Primicenb), Springer-Verlag, 1986.

[3] J. Bear, Dynamics of Fluids in Porous Media, 2nd Edition, Elsevier, New York, 1972.

[4] J.B. Bell, C.N. Dawson and G.R. Sirubin, An unsplit higher order Gudonov method for scalar conservation laws in two dimensions, J. Comp. Phys., $\underline{74}, 1-24$, $198 \mathrm{~s}$.

[5] W.J.P. Bosma, S.E.A.T.M. van der Zee and C.J. van Duijn, Plume development of a nonlinearly adsorbing solute in heterogeneous porous formations, Report 94-45, Delft University of Teclınology, 1994.

[6] H. Brezis, L.A. Peletier and D. Terman, $A$ very singular solution of the heat equation with absorption, Arch. Rat. Mech. Anal., 95, No. 3, 185-209, 1986.

[7] D.T. BuRR, E.A. SudickY and R.L. NAFF, Nonreactive and reactive solute transport in three dimensional heterogeneous porous media: Mean displacement, plume spreading and uncertainty, Water Res. Res., 30, No. 3, 791-815, 1994. 
[8] G. Dagan AND V. CVETKovic, Spatial moments of a kinetically sorbing plume in a heterogeneous aquifer, Water Res. Res., 29, No. 12, 4053-4061, 1993.

[9] C.N. DAwson, Gudonov-mixed methods for advection-diffusion equations in multidimensions, SIAM J. Numer. Anal., $\underline{30}$, 1315-1332, 1993.

[10] C.N. DAwson, Analysis of a upwind-mixed finite element method for nonlinear contaminant transport equations, To appear, 1993.

[11] DiBenidetro, Cintinuity of Weak Solutions to a General Porous Medium Equation, Ind. U. Math. J., 32, No. 1, 83-118, 1983.

[12] C.J. VAN DUIJ AND P. KNABNER, Travelling waves in the transport of reactive solutes through porous media: Adsorption and binary ion exchange, Part I, Transport in Porous Media, $\underline{\mathrm{S}}, 167-194,1992$.

[13] C.J. van Duijn, P. Knabner and S.E.A.T.M. van der Zee, Travelling waves during the transport of reactive solute in porous media: combination of Langmuir and Freundlich isotherms, Advances in Water Res., 16, 97-105, 1993.

[14] M. ESCOBEDO AND E. ZUAZUA, Large time behaviour of convection-diffusion equations in $\mathbb{R}^{N}$, J. Funct. Anal., 100, 119-161, 1991.

[15] M. Escobedo, J.L. VAzQUez AND E. ZUAZUA, A diffusion-convection equation in several space dimensions, Indiana Univ. Math. Journal, $\underline{42}, 1413-1440,1993$.

[16] M. Escobede, J.L. Vazques and E. Zu^zu^, Arch. Rat. Mech. Anal., 124, 43-66, 1993.

[17] R.A. Freeze and J.A. Cilenry, Groundwater, Prentice-Hall, Englewood Cliffs, 1979 .

[18] B.H. Gilding, Improved Theory for a Nonlinear Degenerate Parabolic Equation, Ann. Scuola, Norm. Sup. Pisa Cl. Sci., 16, 165-224, 1989.

[19] C.H. Giles, D. Smiti AND A. Huiton, A general treatment and classification of the solute adsorption isotherm, I. Theoretical, J. Colloidal Surface Sci., 47, 755-765, 1974.

[20] R.E. Grundy, C.J. van Duijn and C.N. Dawson, Asymptotic profiles with fnite mass in one-dimensional contaminant transport through porous media: the fast reaction case, Quart. J. Mech. and App. Maths., 47, Part I, 1994.

[21] R.E. GRUNDY, The asymptotics of extinction in nonlinear diffusion reaction equations, J. Aust. Math. Soc., Ser. B 33, 414-429, 1992.

[22] E.C. Titcilmansil, Eigenfunction expansions associated with second order differential equations, Oxford University Press, 1946. 
[23] W.J. Weber, P.M. MCGinley and L.E. Katz, Sorplion phenomena in subsurface systems: Concepts, models and effect on contaminat fale and transport, Water Res., 2.5, 499-528, 1991. 
